\title{
AC loss modelling and measurement of superconducting transformers with coated-conductor Roebel-cable in low-voltage winding
}

\author{
Enric Pardo ${ }^{1}$, Mike Staines ${ }^{2}$, Zhenan Jiang $^{2}$, Neil Glasson ${ }^{3}$ \\ ${ }^{1}$ Institute of Electrical Engineering, Slovak Academy of Sciences, \\ Dubravska 9, 84104 Bratislava, Slovakia \\ ${ }^{2}$ Robinson Research Institute, Victoria University of Wellington, \\ Wellington, New Zealand \\ ${ }^{3}$ Callaghan Innovation, Christchurch, New Zealand
}

\begin{abstract}
Power transformers using high temperature superconductor (HTS) Re$\mathrm{BCO}$ coated conductor and liquid nitrogen (LN) dielectric have many potential advantages over conventional transformers. The AC loss in the windings complicates the cryogenics and reduces the efficiency, and hence it needs to be predicted in its design, usually by numerical calculations. This article presents detailed modelling of superconducting transformers with Roebel cable in the low-voltage (LV) winding and a high-voltage (HV) winding with more than 1000 turns. First, we model a 1 MVA 11 $\mathrm{kV} / 415 \mathrm{~V} \mathrm{3}$-phase transformer. The Roebel cable solenoid forming the $\mathrm{LV}$ winding is also analyzed as stand-alone coil. Agreement between calculations and experiments of the 1 MVA transformer supports the model validity for a larger tentative $40 \mathrm{MVA} 110 \mathrm{kV} / 11 \mathrm{kV}$ 3-phase transformer design. We found that the $\mathrm{AC}$ loss in each winding is much lower when it is inserted in the transformer than as stand-alone coil. The AC loss in the 1 MVA and 40 MVA transformers is dominated by the LV and HV windings, respectively. Finally, the ratio of total loss over rated power of the 40 MVA transformer is reduced below $40 \%$ of that of the 1 MVA transformer. In conclusion, the modelling tool in this work can reliably predict the $\mathrm{AC}$ loss in real power applications.
\end{abstract}

\section{Introduction}

ReBCO coated conductors are the most promising superconductors (HTS) for power and magnet applications. Many of these applications contain windings with a high number of turns, such as wind generators, motors, transformers, su- 
perconducting magnetic energy storage, and high-field magnets [1-3]. In these applications, changing magnetic fields create AC loss, complicating the cryogenics and reducing the efficiency [4.

Power transformers using high current density HTS wire and liquid nitrogen (LN) dielectric have many potential advantages over conventional oil immersed transformers. They can be smaller, lighter, more efficient, with low fire and environmental hazard and can have overload capability without reduction in lifetime, low output impedance, and fault current limiting capability [5]. More than a dozen HTS transformer demonstrators with ratings of $500 \mathrm{kVA}$ or greater have been built worldwide over a period of more than 15 years. Reported AC loss results (after allowing for cryocooler efficiency) have mostly been around, and in some cases significantly lower than, the $1 \%$ load loss typical of conventional transformers of 1 MVA rating [6]. Of course, AC loss depends sensitively on the ratio of operating current to conductor critical current, and this is not always reported. Using a Roebel cable for the low-voltage high-current winding reduces the $\mathrm{AC}$ loss due to the perpendicular and parallel magnetic field up to a factor 2 and around 1000, respectively 7.

Numerical modelling of the $\overrightarrow{A C}$ loss in transformer windings is necessary for their design. In addition, qualitative properties are still mostly unknown, such as the $\mathrm{AC}$ loss distribution within the winding. Of special relevance is the behaviour of solenoids made of Roebel cable, which have not been previously investigated. The high-voltage windings contain a high number of turns that in some cases is higher than 1000.

Modelling of ReBCO coated conductor coils has been the subject of extensive work 8 15. However, detailed modelling of coils (i.e. modelling the current density in each turn) with more than 1000 turns have not been published. For this case, published work reduces the problem complexity by approximating pancake coils with more than 100 turns as a continuous object 12 16, 17]. However, this is not useful for solenoids or stacks of pancakes with $\sim 10$ turns per pancake. For this purpose, the neighbour approximation has been introduced in [16] but stacks of only up to 32 pancakes have been published.

Roebel cables can be modelled with a cross-sectional 2-dimensional (2D) approximation [7,18. Alghough 3-dimensional (3D) models (or models for thin tapes with 3D bending) provide a more detailed description [19 21, 2D models agree with measurements 20, except for very low alternating current or applied field amplitudes. In addition, 3D computations for Roebel cable coils would require huge computational power and may not be even feasible. At present, only $2 \mathrm{D}$ calculations of pancake coils up to 13 turns and 130 strands have been published 22. Therefore, the qualitative behaviour of solenoids of Roebel cables remains mostly unknown.

This article presents detailed modelling of fully superconducting transformers with more than one thousand turns or Roebel-cable strands in both the high-voltage (HV) and low-voltage (LV) windings. First, we model a 1 MVA $11 \mathrm{kV} / 415 \mathrm{~V}$ 3-phase transformer with a LV winding consisting of Roebel cable solenoid and a HV winding made of a stack of pancake coils (see figure 1 and tables 1 and 22 23 25. Special attention is given to the Roebel cable solenoid, 
which is also investigated as a stand-alone coil. Agreement between model and experiements of the $1 \mathrm{MVA}$ transformer supports the model validity for a larger tentative 40 MVA $110 \mathrm{kV} / 11 \mathrm{kV}$ 3-phase transformer design (tables 1 and 3).

This paper is structured as follows. Section 2 presents the experimental method, measurement results, and the parameters of the measured 1 MVA transformer. Section 3 outlines the model, based on the Minimum Magnetic Energy Variation (MMEV), and the assumptions taken. Afterwards, the article analyses the model results for the current density, magnetic field, AC loss and loss per pancake for the Roebel cable solenoid as stand-alone coil (section 4), the 1 MVA transformer (section 5) and the 40 MVA transformer (section 6). Section 5 also discusses the comparison with experiments for the 1 MVA transformer. Finally, section 7 summarizes our conclusions.

\section{Experiments}

This section presents the parameters of the measured transformer (section 2.1), the critical current of the tape in the transformer windings (section 2.2), and the $\mathrm{AC}$ loss measurement method and results (section 2.3).

\subsection{MVA $11 \mathrm{kV} / 415 \mathrm{~V}$ 3-phase transformer}

The experimental study centers on the 1 MVA 11 kV/415 V 3-phase transformer prototype developed by the Robinson Research Institute and partner organisations (see figure 1 top) and parameters in table 123 25. The low-voltage winding (LV) consists of a 15-strand Roebel cable made from SuperPower tape [26], with $5 \mathrm{~mm}$ width strands, and a $2 \mathrm{~mm}$ central gap between strands. The center and bottom pictures of figure 1 show the structure of the LV winding. This transformer is designed to operate at $70 \mathrm{~K}$, instead of liquid nitrogen temperature at atmospheric pressure, around $77 \mathrm{~K}$. The reason is primarily to increase the current capacity of the Roebel cable but it also has the effect of suppressing boiling in the liquid nitrogen which might reduce the breakdown voltage of the windings.

\subsection{Critical current and critical current density}

The critical current of each strand composing the Roebel cable of one LV winding of the 3-phase transformer has been characterized by self-field transport measurements, and the critical current of short samples of each strand was measured under applied magnetic fields up to $200 \mathrm{mT}$. The angular dependencies for $70 \mathrm{~K}$ and 100 and $200 \mathrm{mT}$ show very different characteristics for 3 groups of strands: low, mid and high. The proportion of the strands were: 3 "low", 7 "mid", and 5 "high" 24. We also measured the magnetic field dependence of the "mid" strands under perpendicular field (see figure 3).

For the 1 MVA 3-phase transformer, in this article we focus on phase "1" 24. The critical current of the Roebel cable for the LV winding at $77 \mathrm{~K}$ have been 

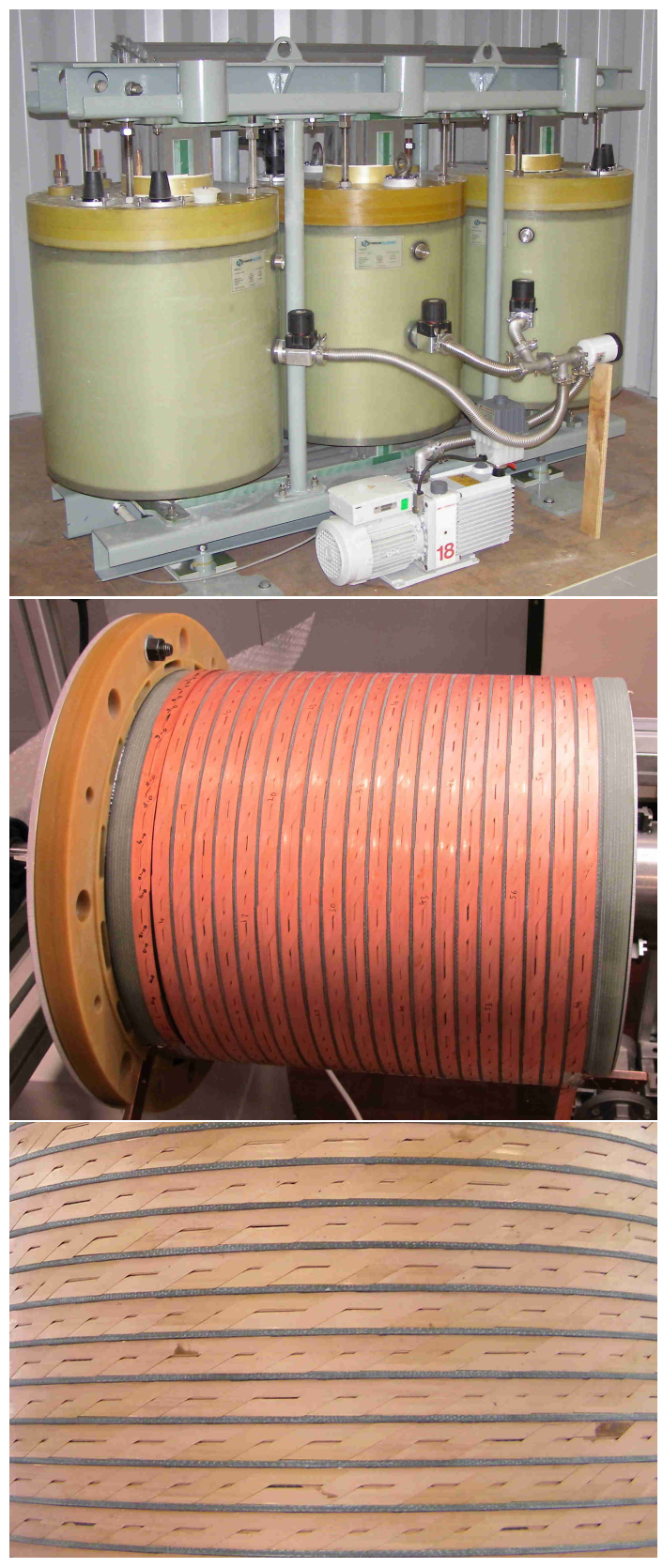

Figure 1: Top: 1 MVA $11 \mathrm{kV} / 415 \mathrm{~V}$ 3-phase transformer 23 25; for clarity, the stage before installing iron cores, current leads and cryo-coolers is shown. Center: LV winding made of Roebel cable. Bottom: Details of the Roebel cable winding. 


\begin{tabular}{lrrr}
\hline \hline & 40 MVA & 1 MVA & ratios \\
\hline Rated power [MVA] & 40 & 1 & 40 \\
LV cable length per phase [m] & 167 & 20 & 8.35 \\
LV winding voltage rms [V] & 6350 & 240 & 26.5 \\
LV rated current amplitude [A] & 2969 & 1964 & 1.51 \\
LV $I_{c}[\mathrm{~A}]$ & 3500 & 3500 & 1 \\
\hline LV $I_{m, \text { rated }} / I_{c}$ & 0.848 & 0.561 & 1.51 \\
\hline $\mathrm{HV}$ wire length per phase [m] & 3041 & 1001 & 3.04 \\
$\mathrm{HV}$ winding voltage rms [V] & 110000 & 11000 & 10 \\
$\mathrm{HV}$ rated current amplitude [A] & 171.4 & 42.9 & 4 \\
$\mathrm{HV} I_{c}[\mathrm{~A}]$ & 194.4 & 194.4 & 1 \\
\hline $\mathrm{HV} I_{m, \text { rated }} / I_{c}$ & 0.882 & 0.220 & 4 \\
\hline \hline
\end{tabular}

Table 1: Electrical parameters of the 40 MVA and 1 MVA transformers, where $I_{m, \text { rated }}$ is the rated current amplitude. The same $I_{c}$ is used for comparison between transformers. When comparing to experiments, the model for constant $J_{c}$ of the 1 MVA transformer assumes $I_{c}=2226 \mathrm{~A}$ and $118.7 \mathrm{~A}$ for the $\mathrm{HV}$ and $\mathrm{LV}$, respectively.

previously measured, with value $1420 \mathrm{~A}$ 24. In this work, we also measured $I_{c}$ at $70 \mathrm{~K}$ for the $\mathrm{LV}$ as stand-alone coil obtaining $2231 \mathrm{~A}$. For these measurements, we used the same technique as for single Roebel cables [24].

\subsection{AC loss measurements}

This section presents the AC loss measurements for single Roebel cables and the windings of phase 1 of the 1 MVA transformer. A detailed comparison of modelling results and measurements is given in section 5.4 .

In figure 4 we compare measurements and modelling for the transport AC loss in a similar Roebel cable to that in the transformer (cable $15 / 5$ in 27]). The agreement is almost perfect for current amplitudes above $0.5 I_{c}$, although the model moderately under-estimates the AC loss at low currents. This shows that the assumed 2D model is useful to predict the AC loss in the Roebel cable, at least at high current amplitudes.

Regarding the transformer, although the full 3-phase transformer contains a ferromagnetic core, the experimental study is done on one single phase with no ferromagnetic core (or with air core), for simplicity. The ferromagnetic core in the full transformer should not significantly influence the AC loss in the superconductor, due to the relatively large distance between the core and the superconductor. The main role of the ferromagnetic core is to reduce flux leakage by increasing the self- and mutual- inductances of the windings. However, for the air-core transformer the leakage flux is already low, achieving a magnetic coupling factor as high as 0.848 (see table 4 for a definition of the magnetic coupling factor and more data on self- and mutual-inductances). In addition, the case of short-circuit LV winding is independent of the mutual- and self- 

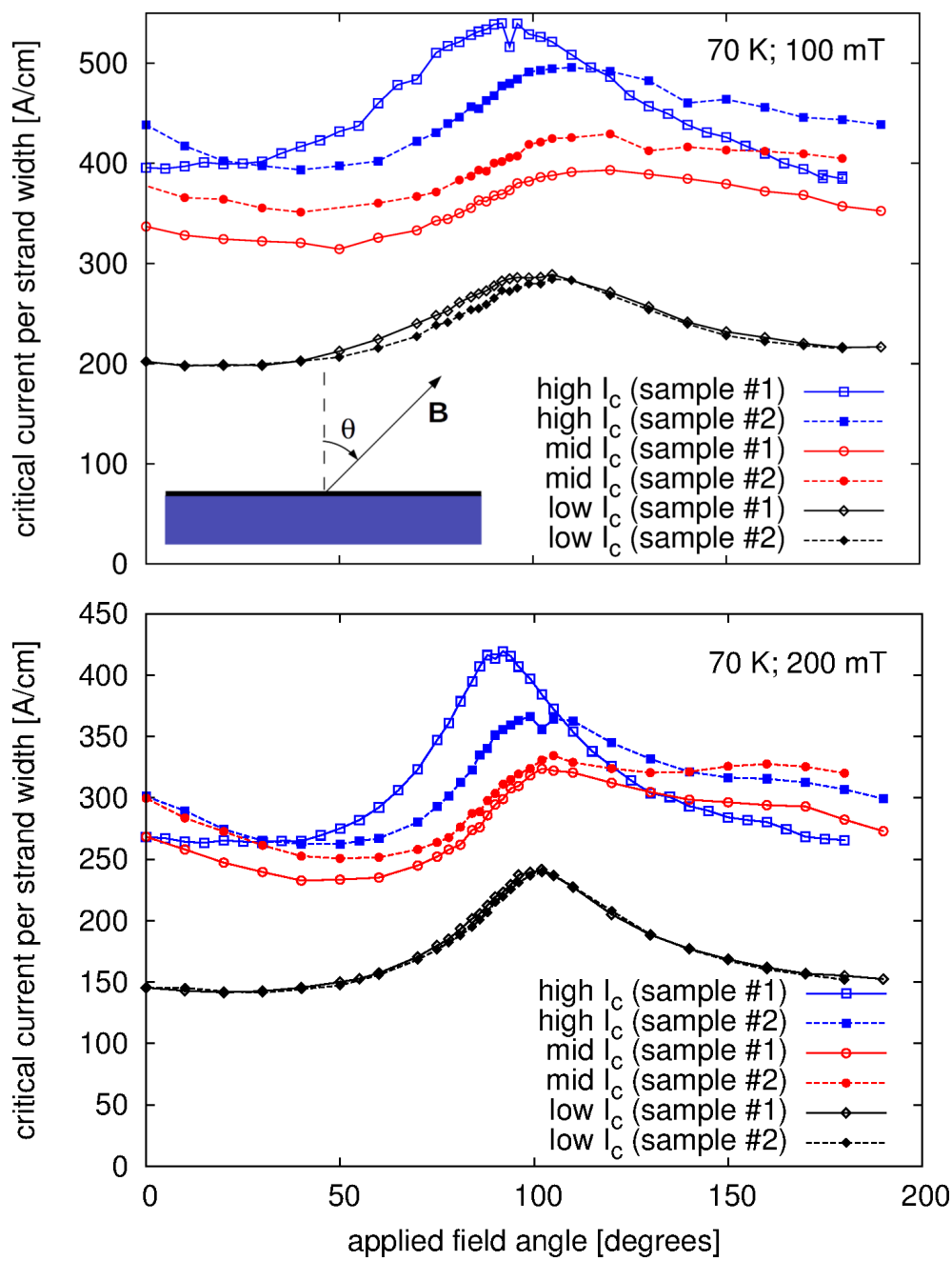

Figure 2: The measured anisotropy of the critical current of the tapes composing the Roebel cable in the LV winding shows three different kinds of characteristics: "low", "mid" and "high". The sketch in the top graph shows the definition of the applied field angle $\theta$. 


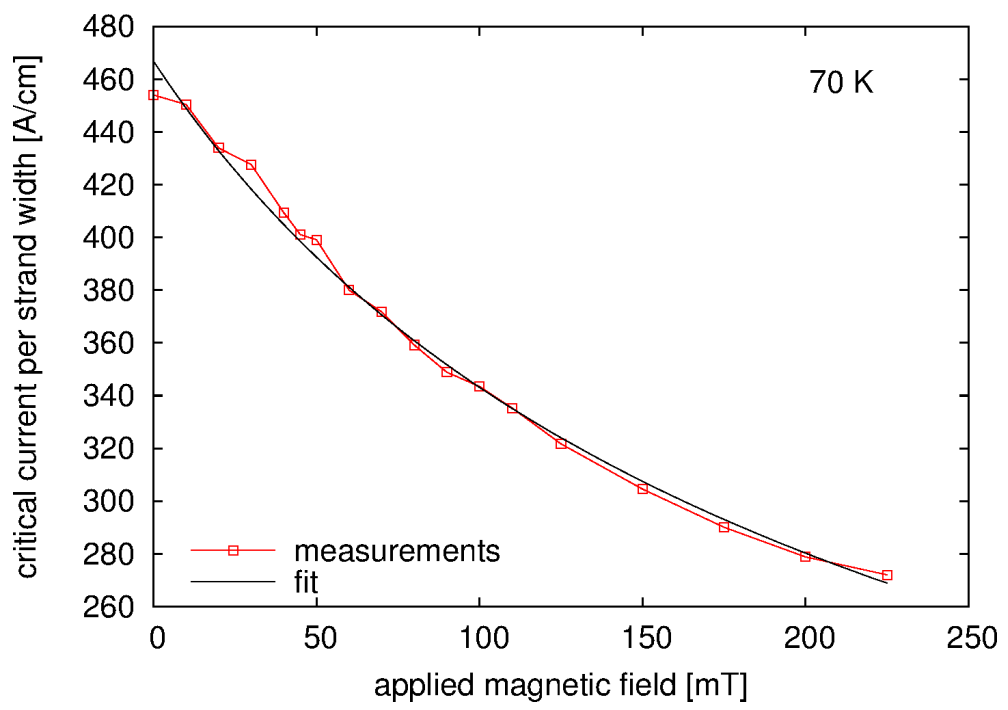

Figure 3: Magnetic field dependence of the "mid" tape with perpendicular applied magnetic field (angle 0 in figure 2).

inductances, as long as the magnetic coupling factor is high. In any case, the higher flux leak in the air-core transformer should increase the AC loss for a given current in the LV winding, since additional current in the HV winding is required to compensate for the flux leakage.

The measured AC loss in the air-core transformer with the LV winding in short-circuit increases with the current amplitude, $I_{m}$, as $I_{m}^{n}$ with $n$ between 4.1 and 2.6 at high and low $I_{m}$, respectively (see figure 5). At the rated current $(1964 \mathrm{~A})$, the $\mathrm{AC}$ loss per phase at $50 \mathrm{~Hz}$ is below $112 \mathrm{~W}$. In addition, for $I_{m}$ close to the rated value or above, the measurements agree with the models. Actually, the measurements were done at $57.08 \mathrm{~Hz}$ and re-normalized to $50 \mathrm{~Hz}$ assuming hysteresis loss, and hence loss proportional to the frequency.

The AC loss was measured on the assembled HV and LV windings in an air-core configuration with shorted secondary. The windings were immersed in a large measurement cryostat, rather than the final transformer cryostats, so that measurements could be made with a low resistance short immersed with the windings in liquid nitrogen. The power delivered to the shorted transformer was measured at the HV terminals and the power dissipated in the short was measured with contacts on the Reobel cable of the LV winding close to the output terminals. The current amplitude and phase were measured with Rogowski coils on the HV inputs and the short. The AC loss of the transformer was obtained from the difference between the input power and dissipation in the short.

The AC loss measurements were made on windings with flux diverters 24 fitted to the end turn of the LV winding. Because AC loss measurements on 


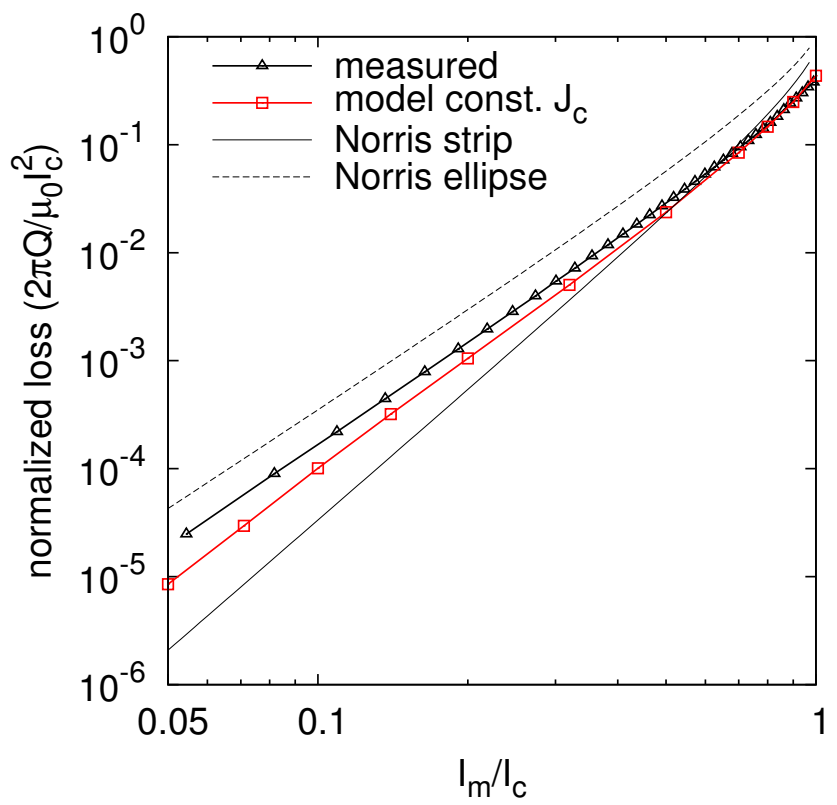

Figure 4: The measured AC loss for a single Roebel cable agrees with the model for constant $J_{c}$, especially for current amplitudes above $50 \%$ of the critical current.

a single LV winding both before and after fitting of the flux diverters showed no measurable change in the $\mathrm{AC}$ loss, in what follows we compare the results of modelling with the flux diverters omitted with the measurements with flux diverters fitted.

\section{$3 \quad$ Numerical method and assumptions}

The results in this report use the Minimum Magnetic Energy Variation (MMEV) method as described in 9, 10. Summarizing, the numerical method takes the sharp current-voltage relation of the critical state model in order to calculate the detailed current density in each turn (or strand of the Roebel cable). Once the current density is known, the instantaneous power loss is computed from the current density and the vector potential that it creates. Afterwards, the loss per cycle is evaluated as twice the time integral of the instantaneous power loss during the last half cycle.

In order to calculate the $\mathrm{AC}$ loss in the $\mathrm{LV}$ and $\mathrm{HV}$ windings of the air-core transformer, we use the following assumptions.

- The critical current density, $J_{c}$, is either assumed constant or with a dependence of the magnetic field, obtained from measurements (see sections 2.2 and 5.3 . 


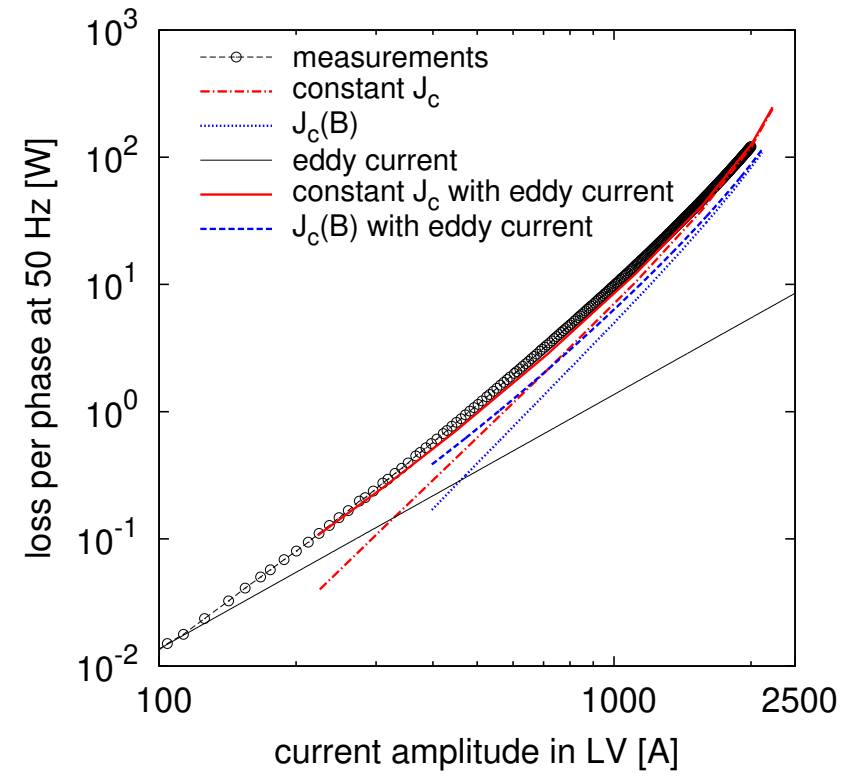

Figure 5: The measured AC loss per unit cycle in the air-core transformer consisting of one phase of the 1 MVA $11 \mathrm{kV} / 415 \mathrm{~V}$ 3-phase transformer agrees with the hysteresis loss models for either constant $J_{c}$ or magnetic-field dependent $J_{c}$, especially at the rated current amplitude (1964 A) and above. 
- The critical current density and its dependence on the local magnetic field is uniform in all tapes and strands. Note, we assume $J_{c}$ to be constant across the width of the conductors, but variation in $J_{c}$ across the width has been linked to AC loss characteristics of commercial tapes 2830 . We have no information about such variation in punched Roebel strand.

- The copper stabilization layer and the metallic substrate present negligible AC loss.

- Axi-symmetrical geometry. This may lead to slight inaccuracies for the LV winding. This is because the LV winding is a helical solenoid and the Roebel cable actually presents a 3D structure. However, it has been shown that the axis-symmetrical approach, which needs to model only the 2D cable cross-section, is a good approximation [31 except for very low applied magnetic fields 20,21. In addition, measurements of the LV winding of the 1 MVA transformer showed a satisfactory agreement with the predictions (see figure 5).

- Constant separation between pancake coils in the HV winding. The HV winding geometry for the 40 MVA transformer has uniform separation between pancake coils, whereas the HV windings of the 1 MVA transformer have a larger spacing between pancakes near the ends of the winding than they do in the middle in order to improve impulse insulation performance.

- When calculating the AC loss in both LV and HV windings, the LV winding is in short circuit. We also assume that the magnetic flux generated by the winding where the $\mathrm{AC}$ loss is not calculated is equivalent to a uniform coil with the same engineering current density and total value of currentturns; the amplitude and phase of the current in that winding is the one arising from the mutual and self-inductances, as described in $\mathrm{A}$. The value of these inductances is given in table 4 and the method to calculate them in B.

\subsection{Parameters in the calculation}

The model takes the dimensions and other parameters of tables 2 and 3 for the 1 MVA and 40 MVA transformers, respectively.

The calculations use between 50 and 400 elements across the tape or strand width (higher values are for lower amplitudes), 1 element in the thickness and a tolerance in the current density $J$ between 0.25 and $0.05 \%$ of $J_{c}$, being the lowest values for the lowest current amplitudes. We checked that these results are neither sensitive to increasing the number of elements across the tape width, nor to decreasing the tolerance percentages. The fact that a single element in the tape thickness is sufficient has been shown in 7 . 


\begin{tabular}{lrr}
\hline \hline & HV & LV \\
\hline Internal diameter [mm] & 345 & 310 \\
No. of turns in axial direction & 48 & 20 \\
No. of turns in radial direction & 19 & 1 \\
Total turns & 912 & 20 \\
Conductor width [mm] & 4 & 12.1 \\
Conductor thickness* [mm] & 0.22 & 0.80 \\
Axial gap between turns [mm] & 2.130 & 2.1 \\
Roebel strand number & - & 15 \\
Strand width [mm] & - & 5 \\
Gap between Roebel stacks [mm] & - & 2.1 \\
Superconducting layer thickness $[\mu \mathrm{m}]$ & 1.4 & 1.4 \\
\hline \hline
\end{tabular}

Table 2: Geometry parameters used for the model of the 1 MVA transformer. (*) Conductor thickness is for individual wire and the cable for the HV and LV windings, respectively. The separation between Roebel strands in the LV winding or turns in the $\mathrm{HV}$ winding are taken from the surface of the superconducting layer. The results for 15 strands are taken as the average between those for 14 and 16 strands.

\begin{tabular}{lrr}
\hline \hline & HV & LV \\
\hline Internal diameter [mm] & 880 & 830 \\
No. of turns in axial direction & 114 & 64 \\
No. of turns in radial direction & 10 & 1 \\
Total turns & 1140 & 64 \\
Conductor width [mm] & 4 & 10 \\
Conductor thickness* [mm] & 0.2 & 0.8 \\
Axial gap between turns [mm] & 2.2 & 1 \\
Roebel strand number & - & 16 \\
Strand width [mm] & - & 4.5 \\
Gap between Roebel stacks [mm] & - & 1 \\
Superconducting layer thickness $[\mu \mathrm{m}]$ & 1.4 & 1.4 \\
\hline \hline
\end{tabular}

Table 3: Geometry parameters of the 40 MVA transformer used in the model. $\left(^{*}\right)$ Conductor thickness is for individual wire and the cable for the HV and LV windings, respectively. The separation between Roebel strands in the LV winding or turns in the HV winding are taken from the surface of the superconducting layer. 


\begin{tabular}{lrr}
\hline \hline & 40 MVA & 1 MVA \\
\hline Self-inductance of the LV $\left(L_{L V}\right)[\mathrm{mH}]$ & 2.58 & 0.0900 \\
Self-inductance of the HV $\left(L_{H V}\right)[\mathrm{mH}]$ & 900 & 219 \\
Mutual inductance $(M)[\mathrm{mH}]$ & 44.1 & 3.76 \\
Magnetic coupling factor $\left(M / \sqrt{L_{L V} L_{H V}}\right)$ & 0.915 & 0.848 \\
\hline \hline
\end{tabular}

Table 4: The calculated inductances reveal that the magnetic coupling factor of both air-core transformers is relatively high. These mutual inductances are calculated as detailed in B

\section{Stand-alone Roebel-cable solenoid: modelling}

In this section, we discuss the model for the standalone LV winding of solenoid shape, without the HV winding. Below, we assume a constant critical current density $J_{c}$ and dimensions in table 2 .

The results of the calculations for a constant critical current density, $J_{c}$, are the following. In these calculations, we took the same critical current as the cable and we assumed a 2D approximation for the Roebel cable (each Roebel cable is made of two parallel stacks of tapes). In this way, we can only take an even number of strands. Actually, the fact that for the studied solenoid the number of strands is odd, with value 15 , does not have an appreciable influence in the analysis in this article. Indeed, as seen in figure 6 the normalized AC loss in the studied Roebel solenoid practically does not change if we take 14 or 16 strands. Although we could take the average between both curves as a more accurate prediction, assuming 14 strands already provides a good loss estimation.

The AC loss of the solenoid made of Roebel cable is qualitatively similar to a Roebel cable in only transport current (figure 7) 18. Moreover, the normalized AC loss per unit cable length in the solenoid made of Roebel cable is only slightly larger than for a single Roebel cable. Actually, the same behaviour appears for solenoids made of one single tape. The AC loss for the complete solenoid falls in between the curves for the Norris strip and ellipse [32]. In general, this is valid for solenoids with many turns, like this one. Previous work showed that for solenoids with few turns (4 to 15 ) the AC loss is usually above Norris' ellipse 33, 34. The reason is that with increasing number of turns, the perpendicular field close to the end turns saturates, while it decreases far away from the ends; as a consequence, the $\mathrm{AC}$ loss per unit conductor length decreases with the number of turns for large turn number. The loss factor ${ }^{1}$ $\Gamma \equiv 2 \pi Q /\left(\mu_{0} I_{m}^{2}\right)$ for the single Roebel cable, where $Q$ is the loss per cycle and cable length, shows that for low current amplitudes it is proportional $I_{m}$ (or $Q \propto I_{m}^{4}$ ), figure 8 . This kind of dependence, typical for thin strips, appears always when the dominant AC loss contribution is from the flux penetration

\footnotetext{
${ }^{1}$ The loss factor $\Gamma=2 \pi Q /\left(\mu_{0} I_{m}^{2}\right)$ is proportioanl to the effective resistance $R_{\text {eff }}$, since $R_{\text {eff }}=2 Q f l / I_{m}^{2}$, where $f$ and $l$ are the frequency and the conductor length, respectively.
} 


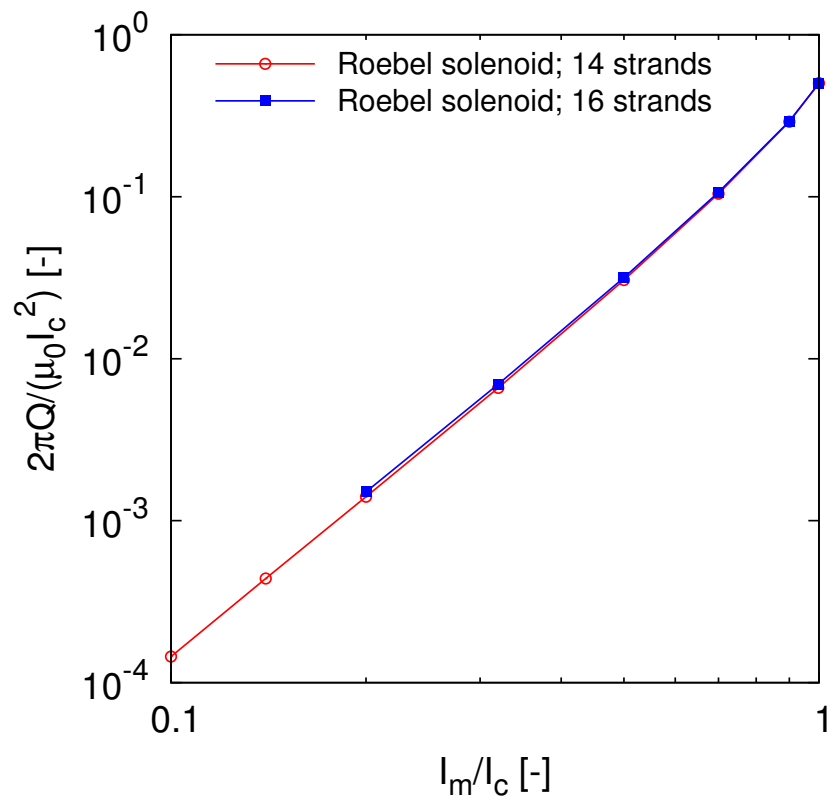

Figure 6: Assuming constant $J_{c}$, the normalized AC loss $2 \pi Q /\left[\mu_{0} I_{c}^{2}\right]$ for a solenoid of Roebel cable with 14 or 16 strands does not depend on the number of strands. $Q$ is the loss per unit cycle and per unit cable length.

from the narrow edges of the tapes 35. With increasing the current amplitude, the behaviour of the Roebel cable approaches to a mono-block, with a loss per cycle proportional to $I_{m}^{3}$. The loss curve for the solenoid made of Roebel cable presents the same qualitative features. In order to calculate the loss for the lowest amplitudes, it was necessary to use 200 and 400 elements in the strands for the Roebel solenoid and the single Roebel cable, respectively.

The AC loss in the solenoid is distributed very inhomogeneously. Figure 9 shows the contribution to the AC loss from each stack of the Roebel cable compared to the total loss. Since the coil has 20 turns, this results in 40 stacks. For an amplitude of the operating current between 0.7 and 0.9 of $I_{c}$, the loss at the end stack is between 4 and 5 times larger than the average loss in the winding.

\section{MVA $11 \mathrm{kV} / 415 \mathrm{~V}$ 3-phase transformer: modelling and measurement}

In order to understand the qualitative behaviour of full superconducting transformers, we discuss in detail the LV winding made of Roebel cable (section 5.1) and the HV winding (section 5.2) for the assumption of constant $J_{c}$. Afterwards, we discuss the effect of $J_{c}(B)$ (section 5.3 and compare with the experiments 


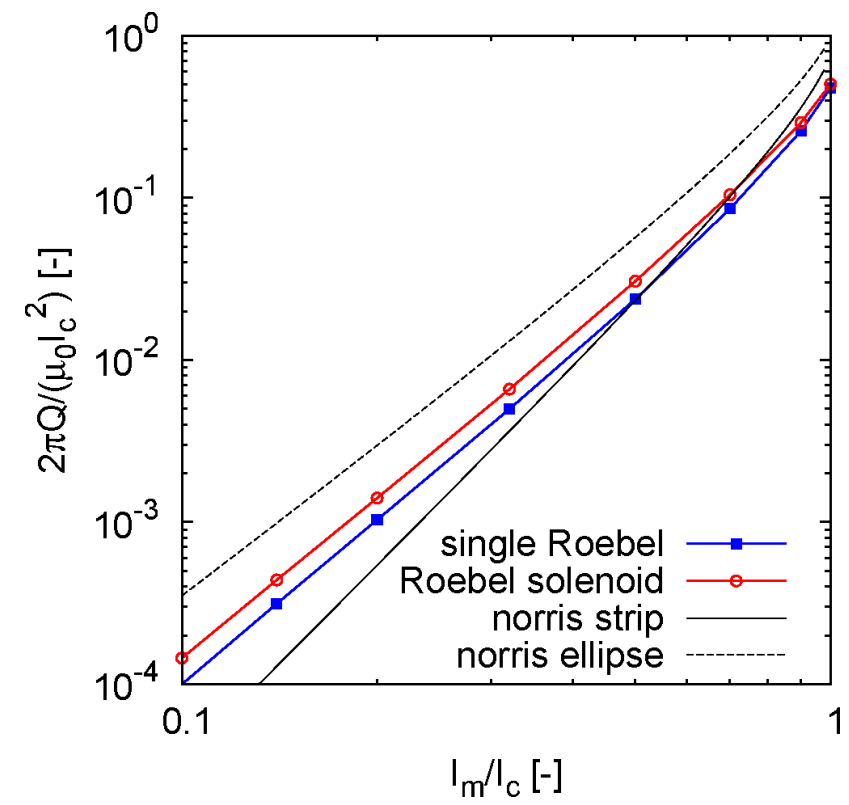

Figure 7: The normalized AC loss per unit cable length in the Roebel solenoid is only slightly higher than for the straigh Roebel cable. The normalized loss is $2 \pi Q / \mu_{0} I_{c}^{2}$, where $Q$ is the loss per cycle and per unit cable length.

(section 5.4.

\section{$5.1 \quad$ LV winding}

The model determines the current in the superconductor assuming that the effective resistance of the superconducting winding is much smaller than its inductive impedance. The effective resistance is defined as the power AC loss, $P$, as $R_{\text {eff }}=2 P / I_{m}^{2}$. Moreover, we assume that the non-linear contribution to the inductance is negligible. We checked that these assumptions are very reasonable by numerical calculations, as follows. The inductance in the LV winding assuming uniform current density proportional to the transport current is $L=89.74$ $\mu \mathrm{H}$. If we assume the real current distribution at the peak of the AC cycle at low amplitudes, $I_{m}=0.1 I_{c}$, the inductance is just $0.5 \%$ smaller (the current distribution for such small current amplitude is already very similar to the Meissner state, and therefore for lower amplitudes there should not be appreciably further deviations of the inductance). Since the current distribution departs the most from a uniform current distribution at low current amplitudes, we can assume that the actual current distribution does not change the inductance, and thence it is constant and given by the value above. The maximum effective resistance at $50 \mathrm{~Hz}$ and $70 \mathrm{~K}$ due to the $\mathrm{AC}$ loss, which appears at the critical current, is around three orders of magnitude smaller than the impedance (the maximum 


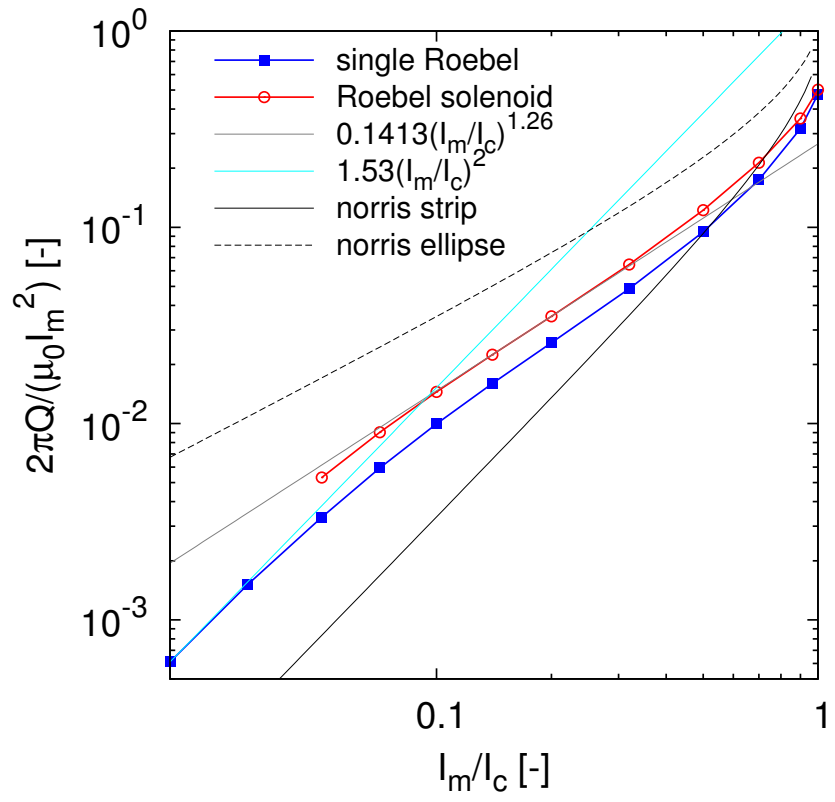

Figure 8: The loss factor $\Gamma=2 \pi Q /\left(\mu_{0} I_{m}^{2}\right)$ for the single Roebel cable, where $Q$ is the loss per cycle and cable length and $I_{m}$ is the current amplitude, shows that for low current amplitudes it is proportional to the current squared (the loss per cycle is proportional to $I_{m}^{4}$ ). The loss curve for the solenoid made of Roebel cable shows the same qualitative features.

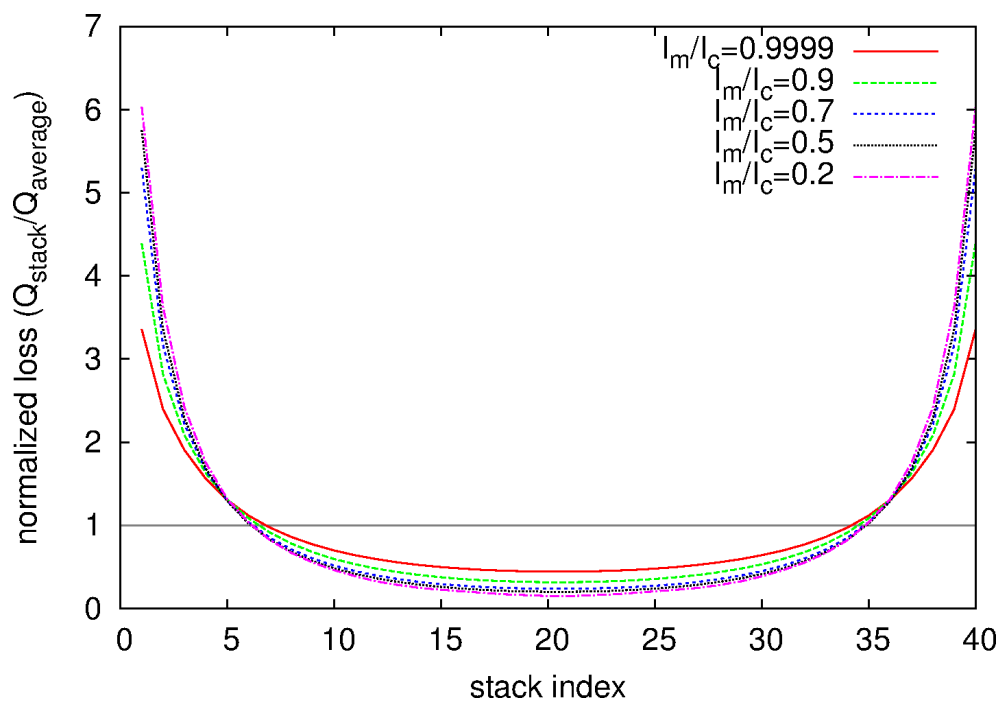

Figure 9: The AC loss in each stack (each Roebel cable is modelled as two parallel stacks of tapes) shows that most of the AC loss in a stand-alone Roebel-cable solenoid appears in the end turns. 
effective resistance is ${ }^{2} 96 \mu \Omega$, while the inductive impedance is $23 \mathrm{~m} \Omega$ ). We can assume, then, that the LV winding is an ideal inductance.

For the transformer, the AC loss in the $\mathrm{LV}$ winding in short circuit (zero load resistance) is much smaller than the $\mathrm{AC}$ loss in the standalone $\mathrm{LV}$ winding (figure 10). The AC loss for the transformer configuration is reduced down to 24, 33 and $41 \%$ of that in the standalone winding for current amplitudes of 20, 70 and $90 \%$ of the critical current. The AC loss falls well below Norris' strip line 32 taking the cable critical current, $I_{c c}$. The AC loss is normalized into the dimensionless quantity $2 \pi Q /\left(\mu_{0} I_{c c}^{2}\right)$, where $Q$ is the loss per cycle and unit cable length. This contrasts with stand-alone windings, where the AC loss per unit tape (or cable) is much larger than the corresponding Norris' strip or ellipse formulas 9, 10. The reason is that in the transformer configuration, the magnetic field from the $\mathrm{HV}$ winding partially cancels the perpendicular magnetic field generated by the LV winding, which is the main cause of the AC loss. This concept has been exploited for fault-current limiters, both resistive 36 39] and inductive [40 41], and also for transformers to a certain extent [42]. Notwithstanding this $\overline{\mathrm{AC}}$ loss reduction, the AC loss for the LV winding is still larger than the Norris' strip for a single strand (or tape).

In the following, we analyse the current density at the peak current of the AC cycle. In particular, we choose a current amplitude $50 \%$ of the critical current, although similar features appear for all amplitudes. For the single solenoid, magnetization currents appear close to the end turns, that is, current density with opposite sign to the transport current (left plot of figure 11). These magnetization currents increase the loss compared to those in the transformer configuration in short circuit where there are no corresponding magnetization currents (figure 12). The absence of magnetization currents is explained as follows. Since the current in the HV winding is perfectly in phase opposition, the perpendicular magnetic field that it creates is in opposite direction to the one from the $\mathrm{HV}$ winding. This results in a decrease in the radial magnetic field in the secondary.

In more detail, the magnetic field in the LV of the transformer is parallel to the conductor except at the end turns and, at a finer scale, also close to the gaps in mid turns as well (see figure 13). In contrast, the magnetic field lines cross the winding region for the solenoid configuration, indicating a larger radial magnetic field (see figure 14). In order to make a quantitative comparison, we consider the average radial magnetic field in each stack of the Roebel cable; defined as $B_{\mathrm{av} r}=(1 / S) \int_{S} \mathrm{~d} S\left|B_{r}\right|$, where $S$ is the stack cross-section (figure 15). At the end of the stacks, the stand-alone coil has 5 times higher average radial field, while the radial field is roughly the same at the central turns. In addition, the average radial field in the transformer configuration is roughly uniform, except at the end turns.

Consequently, the AC loss distribution among the turns (or stacks of strands within the Roebel cable) is much more uniform in the transformer configuration

\footnotetext{
${ }^{2}$ To calculate this value, we use the experimental critical current of the LV winding, 2231
} A. 


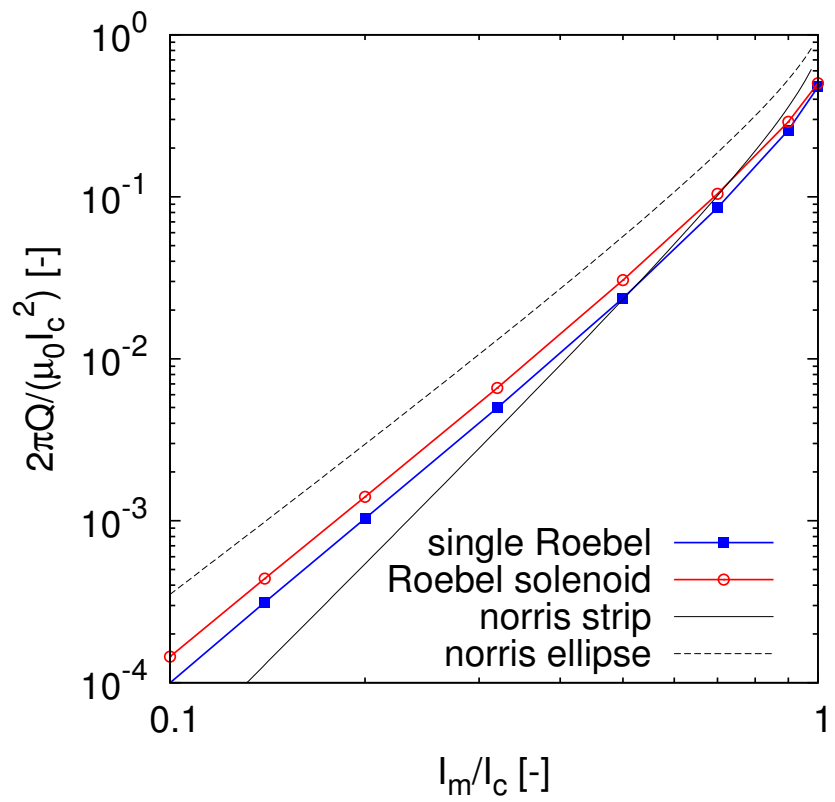

Figure 10: The AC loss in the LV winding in short circuit (zero load resistance) in transformer configuration is much smaller than that as stand-alone coil. The normalized AC loss is below Norris' strip line 32 taking the cable critical current but above the one taking the strand (or tape) critical current. The normalized loss is $2 \pi Q /\left(\mu_{0} I_{c c}^{2}\right)$, where $Q$ is the loss per cycle and unit cable length and $I_{c c}$ is the cable critical current. In the horizontal axis, $I_{c}=I_{c c}$ except for the Norris' strip curve for the strand, where the strand critical current is used. 

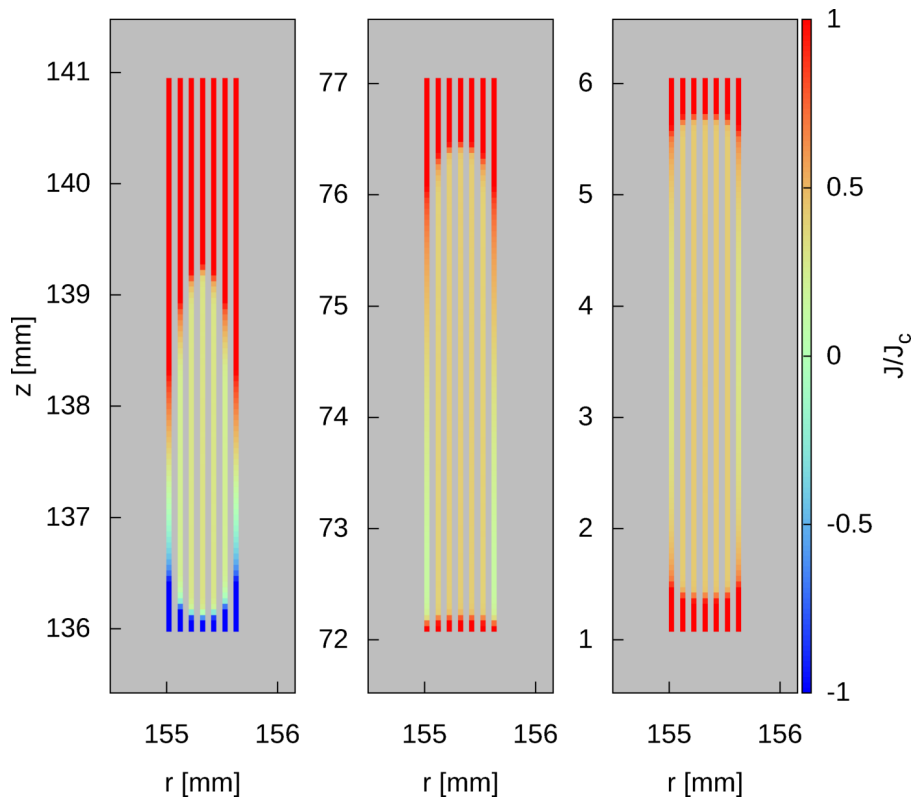

Figure 11: The cross-sections of the stacks within the Roebel cable (half of the Roebel cable) show the current distribution at the top, 3/4 height and center turns of the single solenoid assuming constant $J_{c}$. The instant is the peak of the AC cycle and the current amplitude is $50 \%$ of the critical current. In the graph, the thickness of the superconducting layer is expanded artificially for better visibility (the calculations use the real thickness of the layer). 

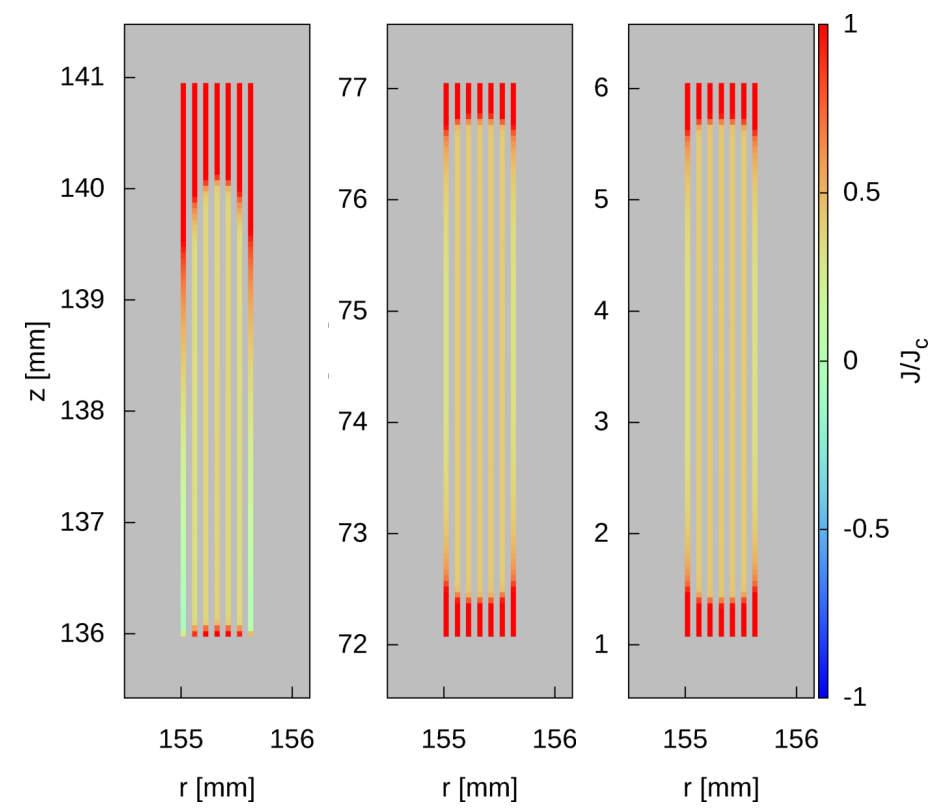

Figure 12: The same as figure 11 but when the solenoid is the LV winding of the 1 MVA transformer. The load resistance is zero (short circuit).

than in the stand-alone solenoid (see figure 16). When the Roebel solenoid is part of the transformer, the distribution of $\overline{\mathrm{AC}}$ loss is a wide plateau for turns far away from the edges. The contribution from each turn (or stack in the Roebel cable) in the plateau is $75 \%$ the average. In contrast, the stand-alone solenoid has a clear U-shape loss distribution with a minimum at the center of around $19 \%$ of the average. This U-shape loss distribution is caused by a similar distribution of the radial magnetic field, which is responsible for most of the AC loss. In the transformer, the $\mathrm{HV}$ winding cancels most of the perpendicular magnetic field in the LV winding, except very close to the ends. The remaining $\mathrm{AC}$ loss in the central turns is due to the residual perpendicular magnetic field due to the finite gap between turns and central gap in the Roebel cable (see figure 13). By reducing the gaps, the AC loss should decrease, although a certain gap is necessary for electric isolation, as well as for the manufacture of the Roebel cable.

To optimize possible coil configurations, it is interesting to explore the correlation between the magnetic field and the AC loss. Since magnetization loss is proportional to a power of the perpendicular magnetic field amplitude with exponent between 3 and 4 , we consider the average $B_{\mathrm{av} r}^{n}=(1 / S) \int_{S} \mathrm{~d} S\left|B_{r}\right|^{n}$ for several exponents $n$, where $S$ is the superconductor cross-section. Next, we compare $B_{\mathrm{av} r}^{n}$ for each stack of the winding and calculate $B_{\mathrm{av} r}^{n}$ either from $B_{r}$ generated by either the numerically calculated $J$ (such as that in figure 12 ) or assuming uniform $J$. To see if power law scaling can give us a quick and 

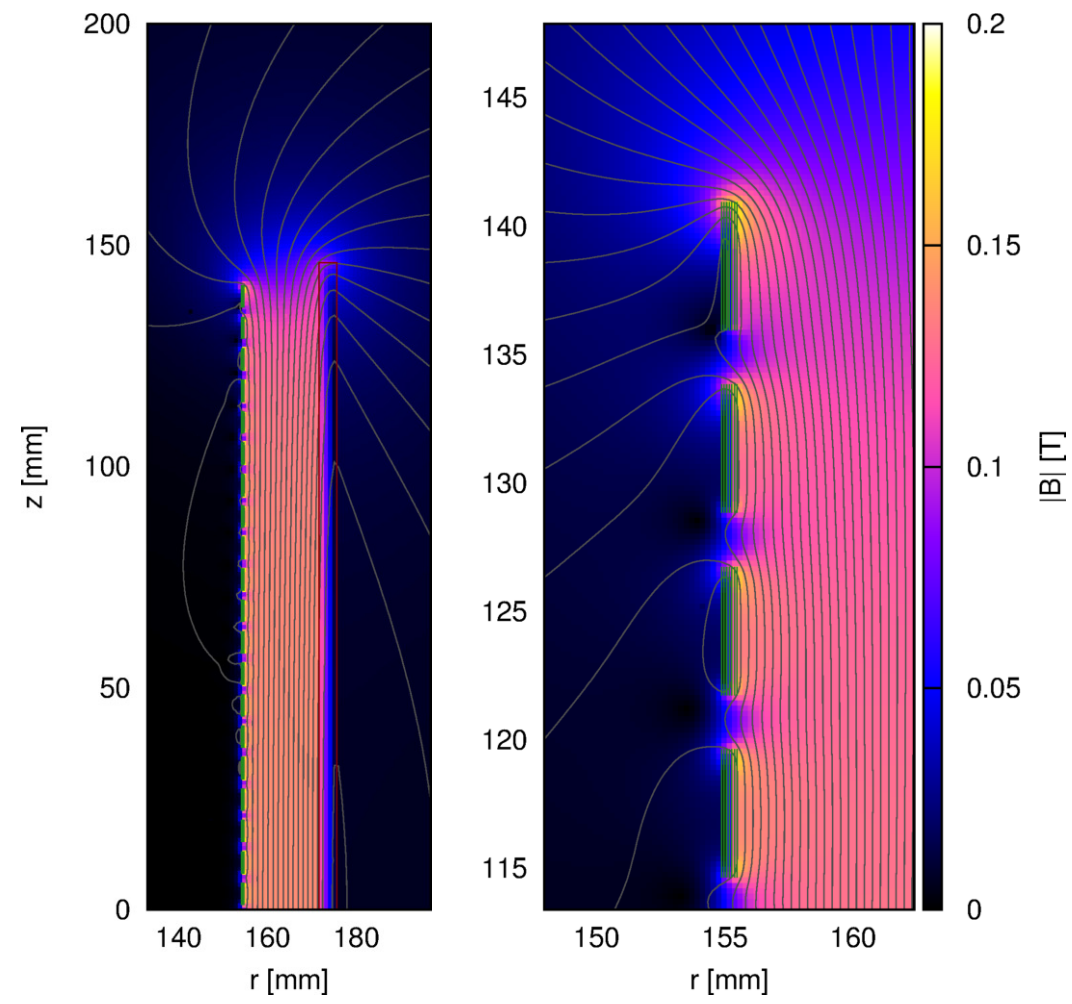

Figure 13: Magnetic field magnitude and magnetic field lines for 1 MVA transformer with the LV winding in short circuit and current amplitude $I_{m}=0.7 I_{c, c}=1562 \mathrm{~A}$ (left: upper half of the transformer; right: detail of the top 4 stacks). The current density in the LV winding is calculated numerically, while we assume constant $J$ in the HV winding for this plot. Green segments represent the cross-section of the LV winding and the red frame represents the HV winding section, the latter approximated as uniform. Magnetic field lines are calculated as curves of constant $r A$. 

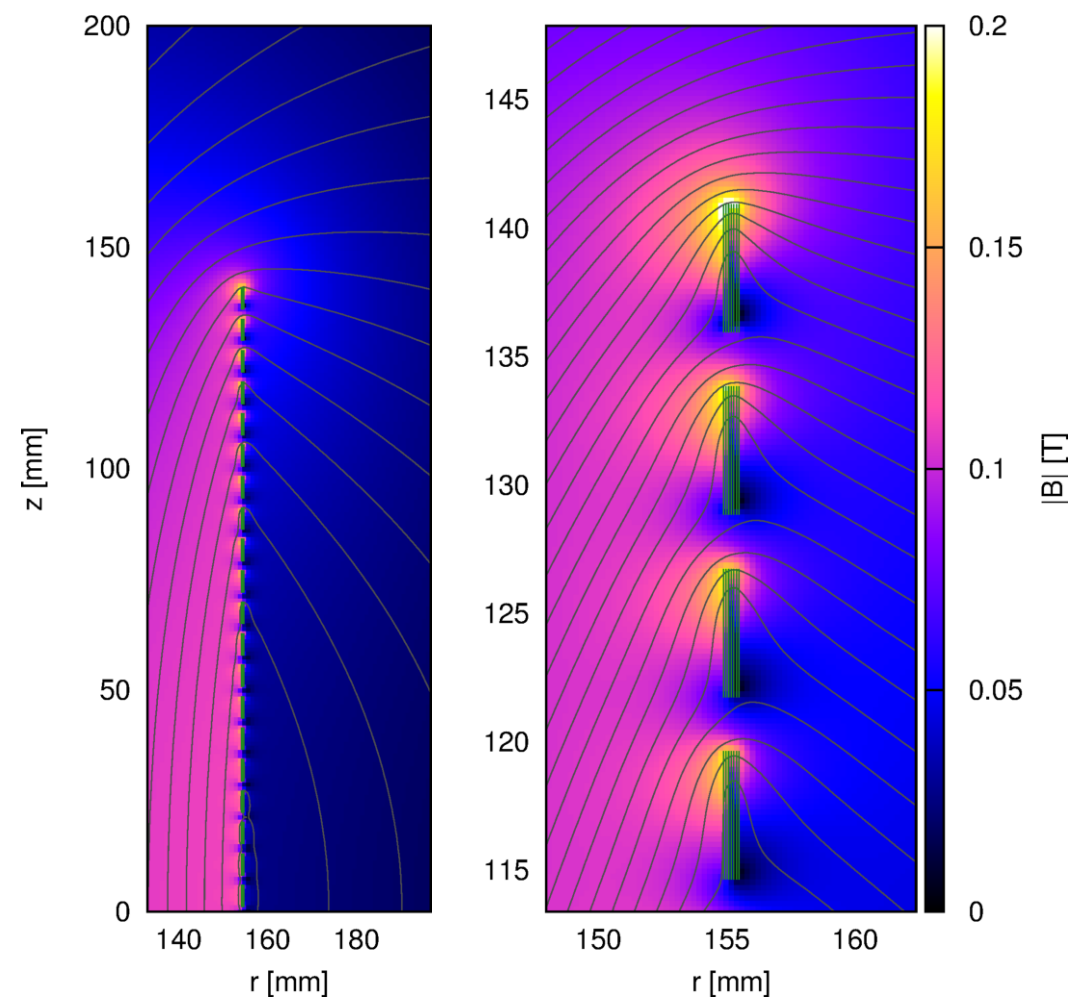

Figure 14: The same as figure 13 but with the LV winding as stand-alone coil (left: upper half of the coil; right: detail of the top 4 stacks). 


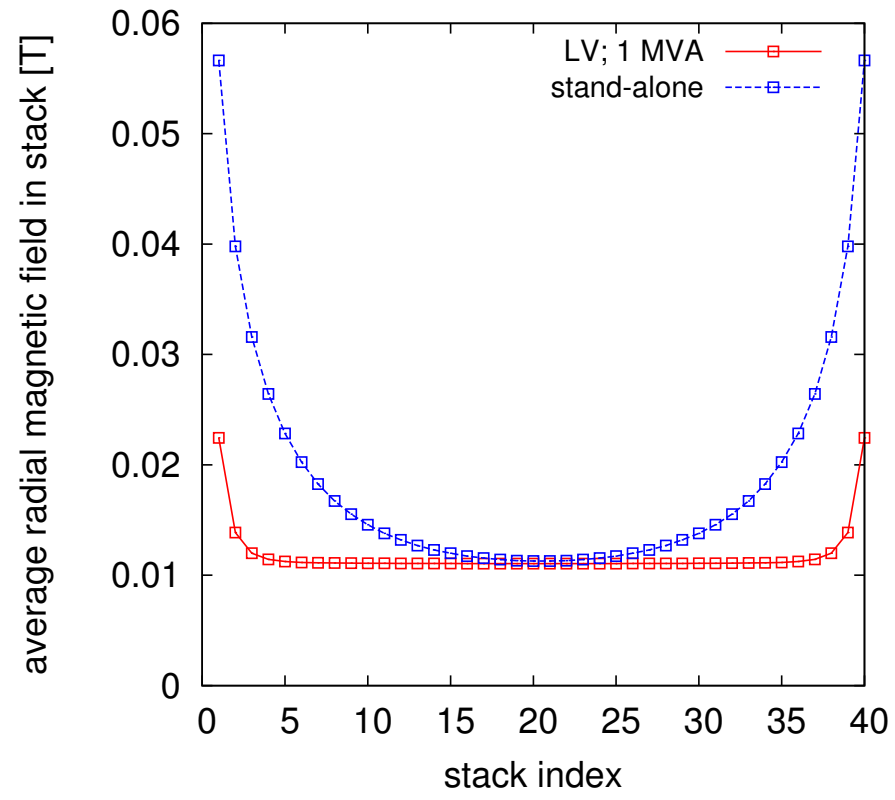

Figure 15: Average magnitude of the radial magnetic field (average of $\left|B_{r}\right|$ ) in each stack of the LV winding for the 1 MVA transformer compared to that of the same winding as stand-alone coil. The current amplitude is $I_{m} / I_{c c}=0.7$, where $I_{c c}$ is the critical current of the Roebel cable; we also assume constant $J_{c}$. 


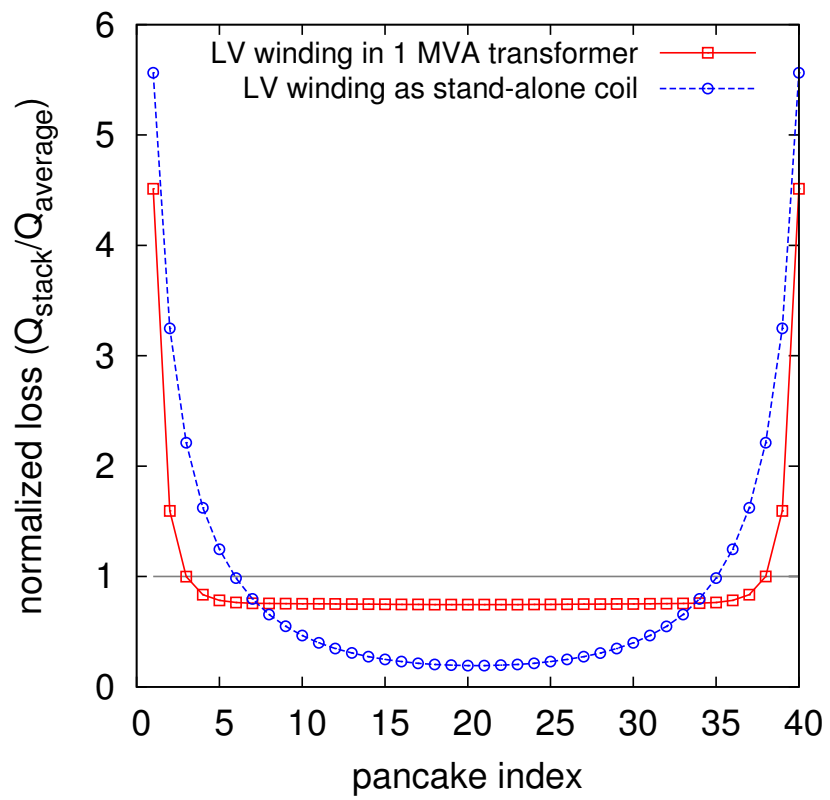

Figure 16: The distribution of AC loss in the LV winding between stacks (Roebel cable approximated as two stacks) in transformer configuration (LV in short circuit) is very different from that as stand-alone solenoid. Calculations for $I_{m} / I_{c c}=0.5$ and constant $J_{c}$. 


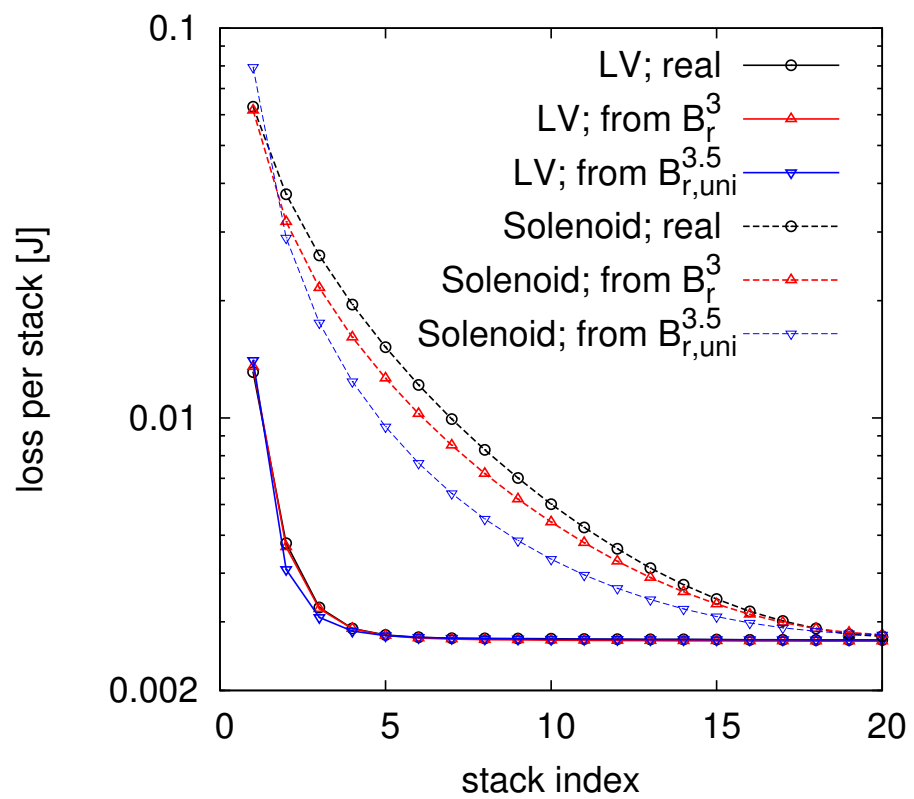

Figure 17: Calculated AC loss per stack in the Roebel cable of the LV winding of the 1 MVA transformer (LV; real) and for the same winding as stand-alone coil (Solenoid; real). The AC loss fairly agrees with the estimations using the average $B_{r}^{3}$ from the

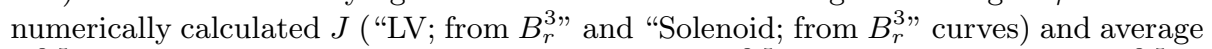
$B_{r}^{3.5}$ computed by assuming uniform $J$ ("LV; from $B_{r, \text { uni }}^{3.5}$ " and "Solenoid; from $B_{r, \text { uni }}^{3.5}$ curves). The numerical calculations assume uniform $J_{c}$.

easy estimate of the $\mathrm{AC}$ loss, we assume that $B_{\mathrm{av} r}^{n}$ is proportional to the $\mathrm{AC}$ loss, and find the proportionality constant $C$ from the total loss $Q$ in the whole coil and the corresponding $B_{\text {avr }}$ as $C=Q S /\left(n_{s} \int_{S} \mathrm{~d} S\left|B_{\text {avr }}\right|^{n}\right)$, where $n_{s}$ is the number of stacks in the winding. The loss in a given stack with index $j$ is then $Q_{j}=C B_{\mathrm{av} r, j}^{n}$. In figure 17 , we can see that the loss distribution in the LV winding practically corresponds to that of $B_{\mathrm{av} r}^{3}$ and $B_{\mathrm{avr} r}^{3.5}$ when using the numerically calculated $J$ and uniform $J$, respectively. There is also a fair agreement for the solenoid when using the same proportionality constants as for the LV winding in the transformer. Therefore, $B_{\mathrm{avr}}^{3.5}$ from uniform $J$ could be used to optimize the winding geometry, greatly simplifying the calculations. This scaling of the local loss averaged over given volumes of the winding as the 3.5th power of the local radial field is consistent with the experimentally determined power law dependence of the total loss with current.

\subsection{HV winding}

The normalized AC loss in the $\mathrm{HV}$ winding when the $\mathrm{LV}$ winding is in shortcircuit is much larger than for a single tape (see figure 18), whether if it is 


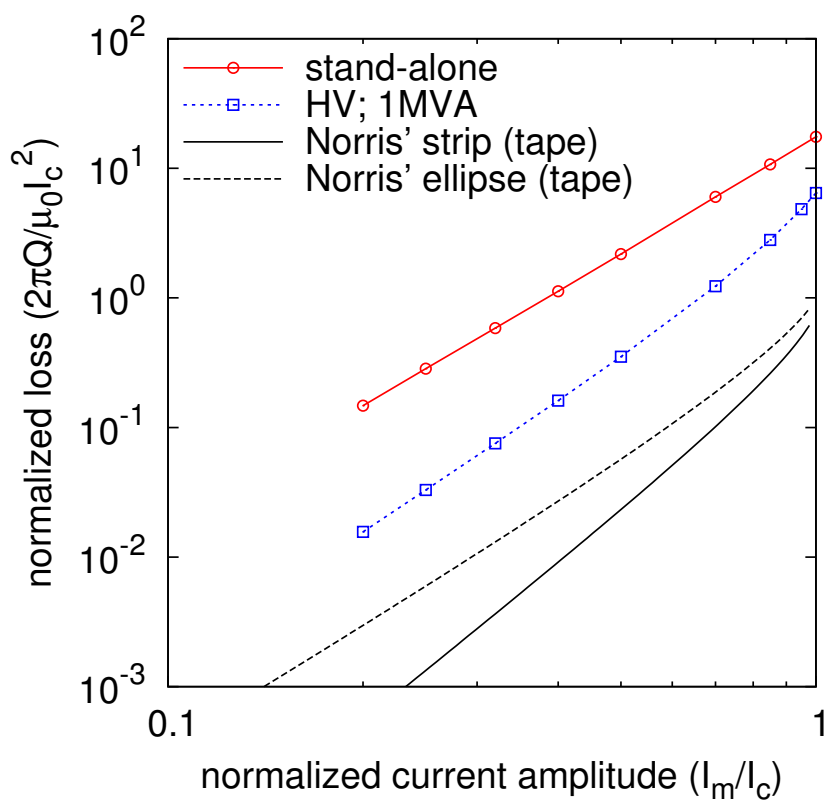

Figure 18: The AC loss in the HV winding with short-circuited LV is much smaller than that of the same coil as stand-alone. Both loss curves fall well above the Norris' strip and ellipse formulas for single tapes 32 .

assumed very thin or elliptic (Norris' thin strip and ellipse formulas 32 ). This is because the neighbouring tapes in the radial direction create a much larger radial field than a single tape, and thence higher AC loss. However, the HV winding in the transformer presents lower AC loss than as stand-alone coil (11, 20 and $29 \%$ of loss in the stand-alone coil for $20,70,90 \%$ of current capacity, respectively).

The cause of this decrease in AC loss is, again, the reduction of the radial magnetic field due to the opposite radial magnetic field generated by the LV winding (see average radial magnetic field in each pancake in figure 19). The magnetic field map and magnetic flux lines in figure 20 show that the magnetic field in the HV winding is parallel to the tape surface except at the four closest pancakes to each end. Close to the top end (figure 20 right) the radial magnetic field is the highest at the top-left corners of the pancakes.

In order to compare the $\mathrm{AC}$ loss between the $\mathrm{LV}$ and $\mathrm{HV}$ windings, we should use the operating conditions of the whole air-core transformer. Figure 21 compares the AC loss in both windings as a function of the current in the LV coil, where we used the relation between the current in both windings from A. The AC loss in the LV winding dominates for all amplitudes. The reason is that for a given current in the LV winding, the 1 MVA transformer design is such that the current in the LV winding relative to its critical one is larger than in the HV winding. For figure 21, we took $J_{c}=2.8393 \times 10^{10} \mathrm{~A} / \mathrm{m}^{2}$ in order 


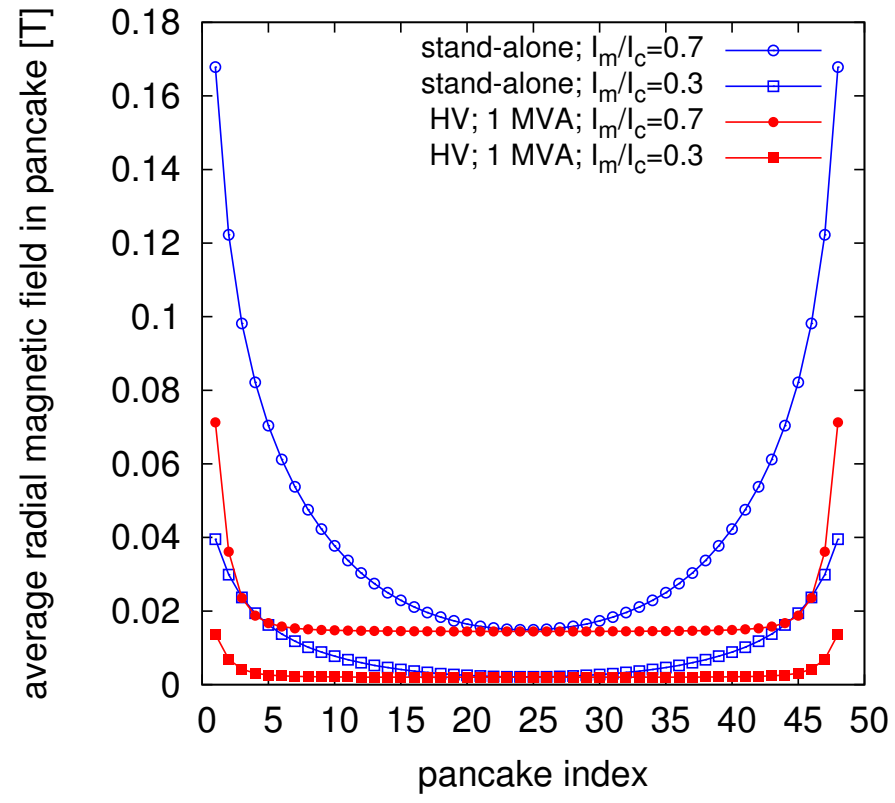

Figure 19: The average radial magnetic field in each pancake of the HV winding is smaller if the winding is part of the transformer (LV in short-circuit) than as standalone coil. $I_{m}$ and $I_{c}$ refer to the current amplitude and critical current, respectively, in the $\mathrm{HV}$ winding. 

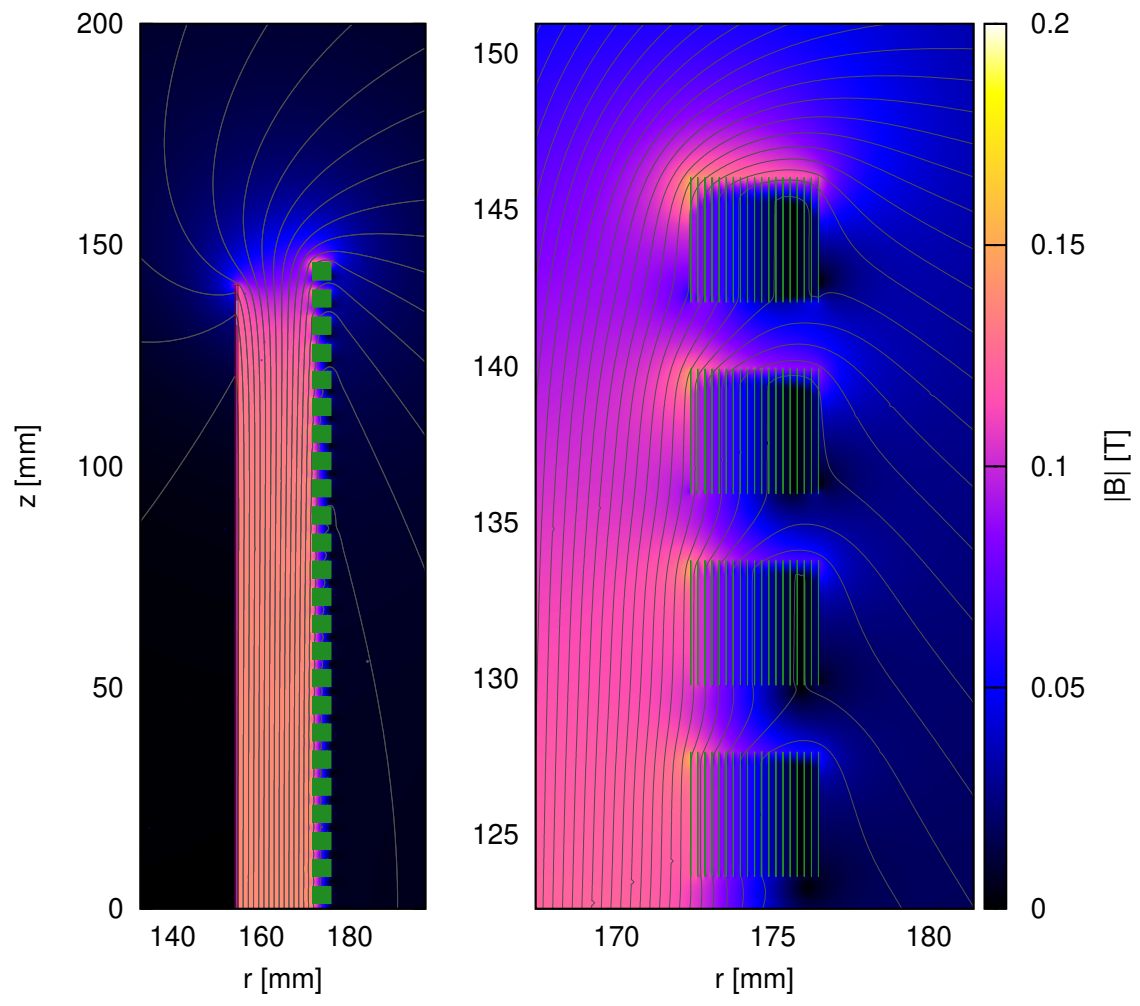

Figure 20: Magnetic field magnitude and magnetic field lines for the same situation as 13 but the $\mathrm{HV}$ is calculated numerically, while the model assumes constant $J$ in the LV. Green segments represent the cross-section of the HV winding and the red frame represents the LV winding section, the latter approximated as uniform. Magnetic field lines are calculated as curves of constant $r A$. 
to compare the results with those for $J_{c}(B)$ and the measurements (see section 5.3 .

\subsection{Effect of $J_{c}(B)$}

As described in section 2, the strands used to manufacture the Roebel cable in the transformer LV windings belong to 3 groups with differing angular dependence of the critical current density. Taking the anisotropy of each strand fully into account in the model is a cumbersome task, specially considering the transposition of the strands in the Roebel cable and that this cable is wound in a coil, forming the LV winding of a transformer. Therefore, we use a simplified isotropic magnetic-field dependence of $J_{c}$. Actually, the anisotropy is relatively weak: $\pm 11 \%, \pm 11 \%$ and $\pm 16 \%$ for the "low", "mid" and "high" families, respectively. The difference in $J_{c}$ between the different families, up to factor two for some orientations ( $\pm 33 \%$ deviation from mid value), is much larger than the difference in $J_{c}$ for different orientations. Therefore, the error caused by using an isotropic $J_{c}(B)$ is much lower than that by assuming identical strands. In addition, commercial tapes present typically a homogeneity of $\pm 10 \%$ of $J_{c}$ along the sample length, reaching $\pm 5 \%$ homogeneity for the very best samples. Therefore, a model describing the anisotropy of $J_{c}$ with an accuracy higher than $\pm 10 \%$ does not necessarily improve the predictions.

Regarding the magnetic field dependence, we took that for the "mid" tape at perpendicular applied field (0 degrees). This orientation approximately falls in the mid value regarding the angular dependence. We found that the magnetic field dependence for this case fits well to a modified Kim model [43,

$$
J_{c}(B)=\frac{J_{c 0}}{\left(1+\frac{B}{B_{0}}\right)^{n}}
$$

where $J_{c 0}, B_{0}$ and $n$ are constants (see fitting curve in figure 3). Afterwards, we scale the whole curve in such a way that $J_{c}$ at $B=0$ equals to the average between all strands of $J_{c}$ in self field for all strands. The parameters of the resulting magnetic field dependence are $J_{c 0}=3.550 \times 10^{10} \mathrm{~A} / \mathrm{m}^{2}, B_{0}=149 \mathrm{mT}$ and $n=0.6$.

The calculated critical current of the LV as stand-alone coil, $I_{\mathrm{C}, \mathrm{S}, \text { calc }}$, agrees with the measured value, $I_{\mathrm{c}, \mathrm{S} \text {, meas }}$ with only $1.6 \%$ difference (see table 5). The model also shows that the inner strand at the end turns limits the critical current, where the magnetic field is the largest (see figure 14). Consistently, $I_{\mathrm{c}, \mathrm{S}, \text { calc }}$ is much smaller than the sum of the strands $I_{c}$ at self-field, $I_{c 0}$ (table 5), because the magnetic field in the coil, $\sim 150 \mathrm{mT}$, is much larger than the strand self-field, $\sim 20 \mathrm{mT}$. Finally, the calculated coil critical current when placed in

the transformer, $I_{\mathrm{c}, \mathrm{LV} \text {, calc }}=2226 \mathrm{~A}$, is practically the same as for the stand-alone situation, $I_{\mathrm{c}, \mathrm{S}, \mathrm{calc}}=2268 \mathrm{~A}$. However, in the transformer configuration the strand limiting the critical current is the outer strand at the central turns, since the average magnetic field in the strand cross-section is the largest (figure 13). 


\begin{tabular}{|c|c|c|}
\hline \multicolumn{3}{|c|}{ Critical current of the LV winding of the 1 MVA transformer } \\
\hline Description & Symbol & Value $[\mathrm{A}]$ \\
\hline Measured as stand-alone coil at $70 \mathrm{~K}$ & $I_{\mathrm{c}, \mathrm{S}, \mathrm{meas}}$ & 2231 \\
\hline Sum of self-field $I_{c}$ of individual strands at $70 \mathrm{~K}$ & $I_{\mathrm{c} 0}$ & 3727 \\
\hline Calculated as stand-alone coil & $I_{\mathrm{c}, \mathrm{S}, \mathrm{calc}}$ & 2268 \\
\hline Calculated as part of the transformer & $I_{\mathrm{c}, \mathrm{LV}, \mathrm{calc}}$ & 2226 \\
\hline
\end{tabular}

Table 5: The calculated critical current using $J_{c}(B)$ from section 5.3 for the LV winding of the 1 MVA transformer as stand-alone coil agrees with the measurements with only $1.6 \%$ difference.

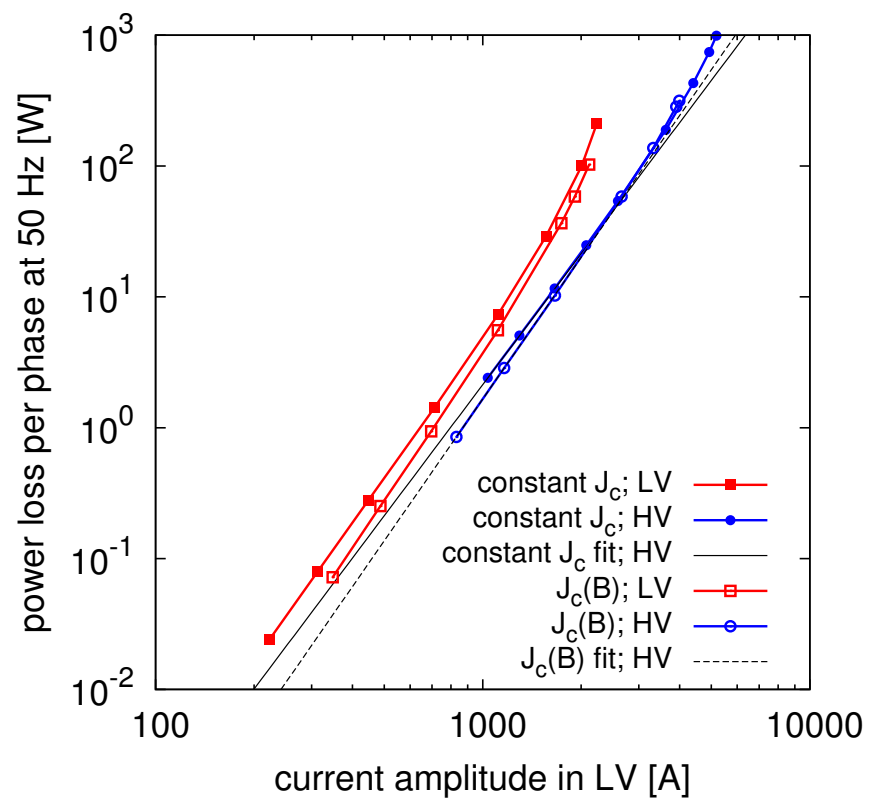

Figure 21: Assuming magnetic-field dependence of $J_{c}, J_{c}(B)$, does not strongly influence the results, compared to the ones for constant $J_{c}$ (computations for the 1 MVA transformer). The lines without symbols are for an analytical fit (power loss proportional to $I_{m}^{n}$ with constant $n$ ) of the AC loss in the LV winding at low current amplitudes. 


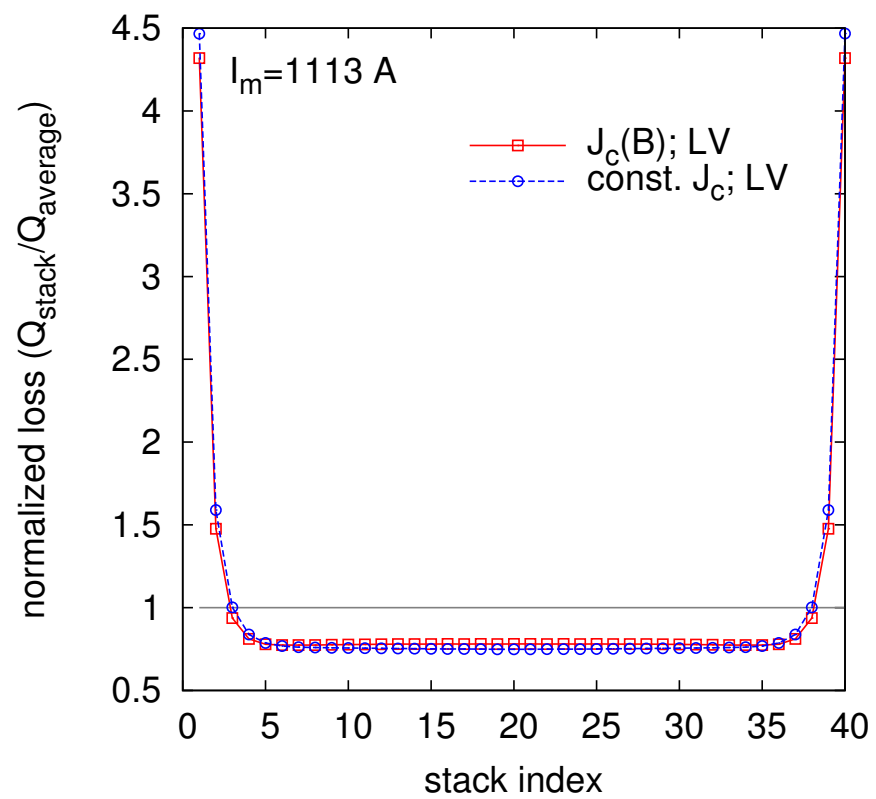

Figure 22: The AC loss distribution between stacks of the LV winding of the 1 MVA transformer practically does not differ between the models with constant $J_{c}$ and magnetic-field dependent $J_{c}, J_{c}(B)$.

In the following, we compare the $\mathrm{AC}$ loss obtained for $J_{c}(B)$ and constant $J_{c}$. For the latter, we take $J_{c}$ as the cross-section average of the local $J_{c}$ at the critical current of the LV,

$$
J_{\mathrm{c}, \mathrm{const}}=I_{\mathrm{c}, \mathrm{LV}, \mathrm{calc}} /\left(n_{s} S_{s}\right),
$$

where $n_{s}$ are the number of Roebel strands in the cable and $S_{s}$ is the strand cross-section, respectively. The AC loss contribution from each winding, LV and $\mathrm{HV}$, computed with the $J_{c}(B)$ dependence is similar to that calculated with constant $J_{c}$ (see figure 21). However, in the LV winding, the AC loss assuming constant $J_{c}$ is slightly larger than for the $J_{c}(B)$ dependence. The reason is that for $J_{c}(B)$, the critical current of most of the strands is below $I_{\mathrm{c}, \mathrm{LV} \text {, calc }}$, and hence the loss for any $I_{m}$ is lower than for constant $J_{c}$, for which the critical current is $I_{\mathrm{c}, \mathrm{LV} \text {,calc }}$ for all the strands. With decreasing current amplitude, the difference increases because the local $J_{c}$ for $J_{c}(B)$ increases. Regarding the AC loss in the $\mathrm{HV}$ winding, there is a cross-over between both curves; where the predictions for $J_{c}(B)$ are lower for low currents, and opposite for higher currents. The cross-over point corresponds roughly to the critical current of the $\mathrm{LV}, I_{\mathrm{c}, \mathrm{LV} \text {, calc }}$. This is because below this current amplitude the local $J_{c}$ for $J_{c}(B)$ in the HV is higher than $J_{\text {c,const }}$, and opposite otherwise.

The $J_{c}(B)$ dependence practically does not influence the AC loss distribution among the turns in the $\mathrm{LV}$ winding (see figure 22). For the $J_{c}(B)$ dependence, 


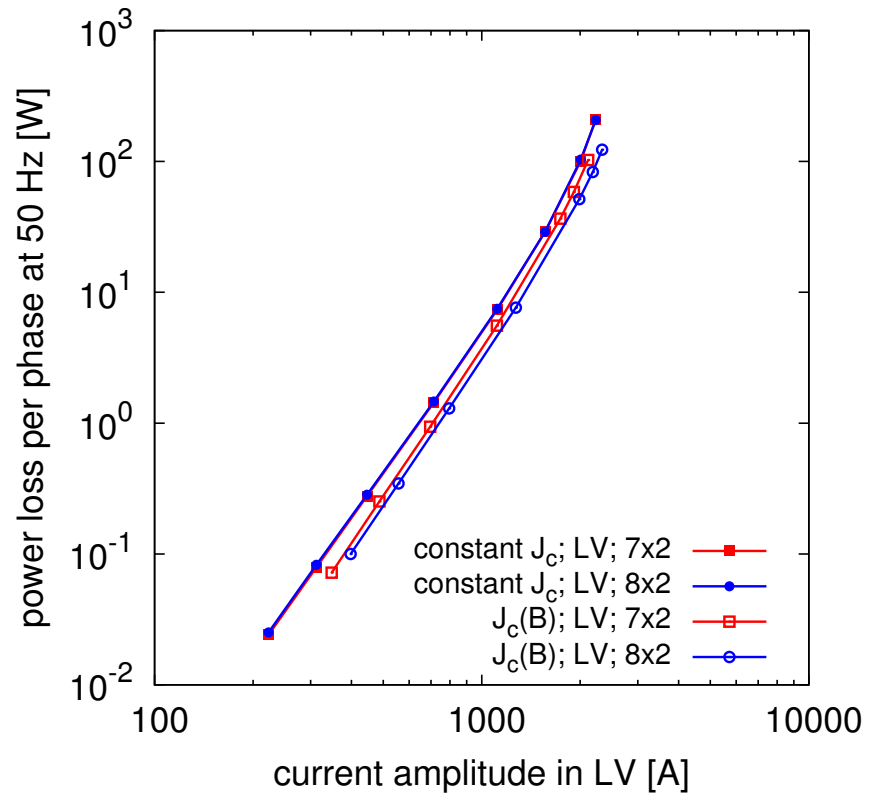

Figure 23: For the model of the 1 MVA transformer with $J_{c}(B)$, taking 14 or 16 stands in the Roebel cable in the LV winding provides moderately different results, while for constant $J_{c}$ the number of strands between this range does not appreciably influence the computations.

there is a slight increase of the relative AC loss close to the coil center. This is caused by the higher magnetic field in that region; decreasing $J_{c}$, and thence increasing the $\mathrm{AC}$ loss.

For a $J_{c}(B)$ dependence, choosing 14 or 16 strands to model the Roebel cable in the LV winding has a small but visible influence on the AC loss, while for constant $J_{c} 14$ and 16 strands provide virtually the same result (see figure 23). The cause is that for $J_{c}(B)$ the critical current density is not normalized to the number of strands, as done for constant $J_{c}$. Therefore, in the comparison with experiments, for $J_{c}(B)$ we used the average between the results for 14 and 16 strands as an estimation of those for 15 .

\subsection{Comparison with experiments}

As shown in figure 5. the approximated $J_{c}(B)$ dependence from section 5.3 already provides a good agreement with experiments close to the rated current amplitude (1964 A) or above. The reason is that the most common strands in the cable are of the type "mid" ( 7 of 15 strands) with only $\pm 11 \%$ variation in $J_{c}$ from angular dependence. In addition, the AC loss scales with $J_{c}$ as $J_{c}^{1 / n}$ with $n$ between around 1.3 and 2 (see figure 34 ). This means that relative deviations of $J_{c}$ from the mid value create an additional loss up to around the same relative 
value. For instance, deviations of $20 \%$ from the mid value in $J_{c}$ create only up to $20 \%$ additional loss compared to those calculated from the mid value. This explains why the predictions of the total loss (loss in LV and HV windings) slightly under-estimates the AC loss compared to the measurements. For low current amplitudes, the distance between the measured and calculated curve with $J_{c}(B)$ increases.

The eddy current loss in the current leads of the LV winding is the main cause of disagreement between model and measurements at low $I_{m}$. This eddy current loss can be estimated as follows. The two copper current leads in the LV winding are placed at the end turns and are plate-shaped with cross-section $d_{r} \times d_{z}$ in the $r$ and $z$ directions, respectively. Assuming the slab approximation, uniform external magnetic field of amplitude $B_{\mathrm{m} r}$ and $B_{\mathrm{m} z}$ in the radial and axial directions, respectively, and that $|\mathbf{B}|$ from the eddy currents is much smaller than the external magnetic field, the eddy current loss per cycle and unit length is

$$
\begin{aligned}
& Q_{\mathrm{e}}=Q_{\mathrm{e} r}+Q_{\mathrm{e} z} \\
& Q_{\mathrm{e} r}=\frac{\pi^{2} f_{\text {meas }} d_{r} d_{z}^{3}}{6 \rho} B_{\mathrm{m} r}^{2} \\
& Q_{\mathrm{e} z}=\frac{\pi^{2} f_{\text {meas }} d_{r}^{3} d_{z}}{6 \rho} B_{\mathrm{m} z}^{2},
\end{aligned}
$$

where $f_{\text {meas }}$ is the measurement frequency and $\rho$ is the copper resistivity. For a current $I=0.1 I_{c}=223 \mathrm{~A}$, the average in the turn cross-section of the magnetic fields in the $z$ and $r$ directions are $B_{\mathrm{av} r}=0.483 \mathrm{mT}$ and $B_{\mathrm{av} z}=7.44$ $\mathrm{mT}$, respectively. $B_{\mathrm{av} r}$ is much smaller than $B_{\mathrm{av} z}$ because for low currents the superconductor shields the radial component. The corresponding coil factors are $b_{r}=B_{\mathrm{av} r} / I=2.16 \cdot 10^{-6} \mathrm{~T} / \mathrm{A}$ and $b_{z}=B_{\mathrm{av} z} / I=3.33 \cdot 10^{-5} \mathrm{~T} / \mathrm{A}$. With these coil factors, $Q_{\mathrm{e} z} / Q_{\mathrm{e} r}=59$, and hence $Q_{\mathrm{e} r}$ can be neglected. Then, the estimated $Q_{\mathrm{e}}$ at low current amplitudes is

$$
Q_{e}\left(I_{m}\right) \approx \frac{\pi^{2} f_{\text {meas }} d_{r}^{3} d_{z}}{6 \rho} b_{z}^{2} I_{m}^{2} .
$$

After adding a contribution proportional to $I_{m}^{2}$ that merges to the measurements at low $I_{m}$, the calculations agree with the measurements for all $I_{m}$ (figure 5). Using that the length of each current lead is $40 \mathrm{~mm}$ and $d_{r}=6 \mathrm{~mm}, d_{z}=12 \mathrm{~mm}$, $f_{\text {meas }}=57.05 \mathrm{~Hz}, \rho=2.40 \cdot 10^{-9} \Omega \cdot \mathrm{m}$, and (5) the eddy current curve in figure 5 corresponds to $b_{z}=2.9 \cdot 10^{-5} \mathrm{~T} / \mathrm{A}$, which is only $13 \%$ below the calculated one above.

Curiously, the hysteresis loss predictions for constant $J_{c}$ from (2) provide closer results to experiments. This is because the over-estimation by taking constant $J_{c}$ partially cancels the under estimation of our approximated $J_{c}(B)$ dependence. 


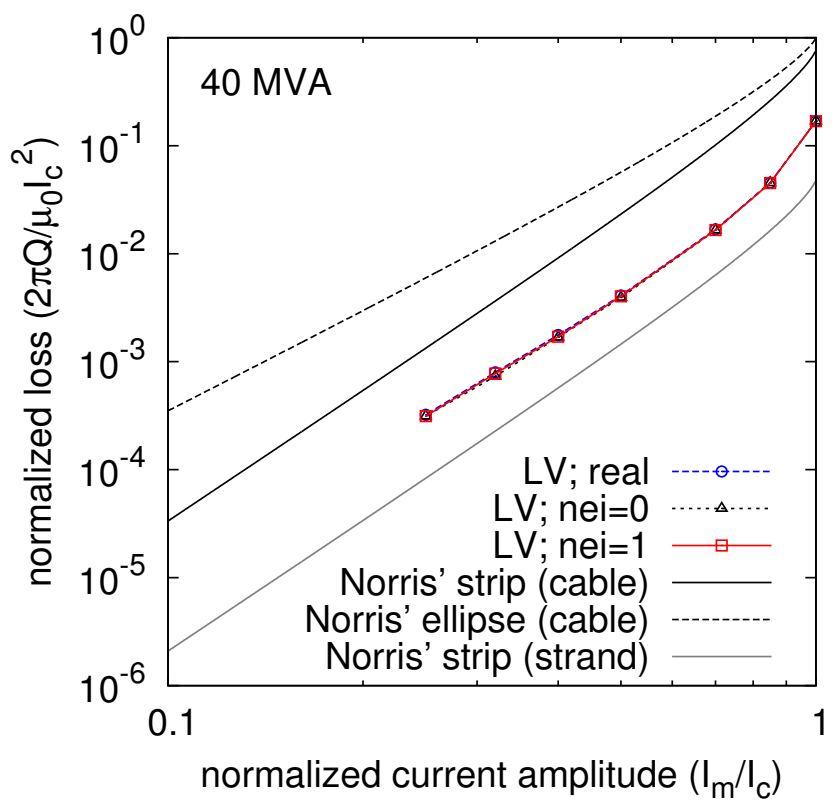

Figure 24: The normalized AC loss in the LV winding in short circuit of the 40 MVA transformer is below Norris' strip line 32 taking the cable critical current but above the one taking the strand (or tape) critical current. The normalized loss is $2 \pi Q /\left(\mu_{0} I_{c c}^{2}\right)$, where $Q$ is the loss per cycle and unit cable length and $I_{c c}$ is the cable critical current. In the horizontal axis, $I_{c}=I_{c c}$ except for the Norris' strip curve for the strand, where the strand critical current is used. Curves for "nei=1" and "nei=0" are for the neighbor approximations of the first and zeroth order, respectively, while "real" takes the full geometry into account.

\section{MVA $110 \mathrm{kV} / 11 \mathrm{kV}$ 3-phase transformer: modelling}

The proposed 40 MVA $110 \mathrm{kV} / 11 \mathrm{kV}$ 3-phase transformer is not simply an upscaling of the 1 MVA $11 \mathrm{kV} / 415 \mathrm{~V} 3$-phase transformer. Therefore, we next discuss the main features of the loss in both the LV winding (section 6.1) and the $\mathrm{HV}$ winding (section 6.2) assuming constant $J_{c}$. Afterwards, we compare the AC loss between the $40 \mathrm{MVA}$ and $1 \mathrm{MVA}$ transformers (section 6.3). Finally, we discuss the dependence of the AC loss with the tape critical current (section 6.4.

\subsection{AC loss in LV winding}

For the 40 MVA transformer, we use the neighbor approximation to speed-up the computing time 16]. For this reason, we checked that the neighbor approximation is valid for our calculations (see figure 24). The approximation taking 
the interaction of the first neighboring turns ("nei=1" curve in figure 24) provides practically the same results as the full model ("real" curve in figure 24). Actually, neglecting the interaction of magnetization currents between neighbor turns is already satisfactory ("nei=0" curve in figure 24). In the following, we present results for either the full geometry or the neighbor approximation of first order without distinguishing between them, unless stated otherwise. In general, the difference between the results for the neighbor approximation of the first and zeroth order provides a good error estimation of this kind of approximation. Computations for the 40 MVA transformer show that the total CPU time is around 15 and 4 times lower for neighbor approximations of the 0th and 1st order, respectively. Tests with the number of turns in the axial direction reduced by one half or one fourth resulted in roughly the same proportional reduction in CPU time. Since the numerical routine for the neighbor approximation is parallelized, the elapsed time using an 8-thread processor (in our case, an Intel(R) Core(TM) i7-4770K) is 120 and 36 times lower for 0th and 1st order neighbor approximation, respectively. The elapsed time for one current amplitude was around 6 hours and $20 \mathrm{~min}$ for the 0th order neighbor approximation.

The AC loss in the LV winding in short circuit of the 40 MVA transformer (figure 24) falls well below Norris' strip line taking the cable critical current [32, although it is still above the Norris' strip curve for a single strand (or tape).

As for the 1 MVA transformer, the AC loss in each turn is the highest at the top and bottom turns (figure 25 but there is a substantial AC loss contribution from the central turns. The $\overline{\mathrm{AC}}$ loss per stack for those far away from the ends is of $64 \%$ and $50 \%$ of the average and the closest 2 turns to each end account for $38 \%$ and $45 \%$ of the total AC loss for a current amplitude of 70 and $50 \%$ the critical current, respectively.

\subsection{AC loss in $\mathrm{HV}$ winding}

As for the LV winding, the AC loss per unit tape length for the HV winding (taking the LV in short circuit) is higher than the Norris' formula for a single tape (see figure 26). However, we expect that the AC loss for the transformer layout is much lower than for a stand-alone coil. In addition, the neighbor approximation [16 taking first neighbors gives practically the same results as that taking no neighbors (figure 26), and therefore the neighbor approximation does not introduce errors in the calculations.

Comparing the $\mathrm{AC}$ loss in the $\mathrm{LV}$ and $\mathrm{HV}$ (figure 27), the $\mathrm{AC}$ loss in the $\mathrm{HV}$ is higher by a factor around 2.5 at $70 \%$ of the current capacity of the LV winding. This comparison has been done for the same current amplitude in the LV winding, using equation (7) for the relation between the current in the HV and LV windings.

The relative AC loss in each pancake (figure 28) shows that, although the $\mathrm{AC}$ loss in the top and bottom pancakes of the $\mathrm{HV}$ winding is much larger than the average, most of the AC loss is generated by the pancakes away from the ends. This suggests that the AC loss due to the radial magnetic field at the 


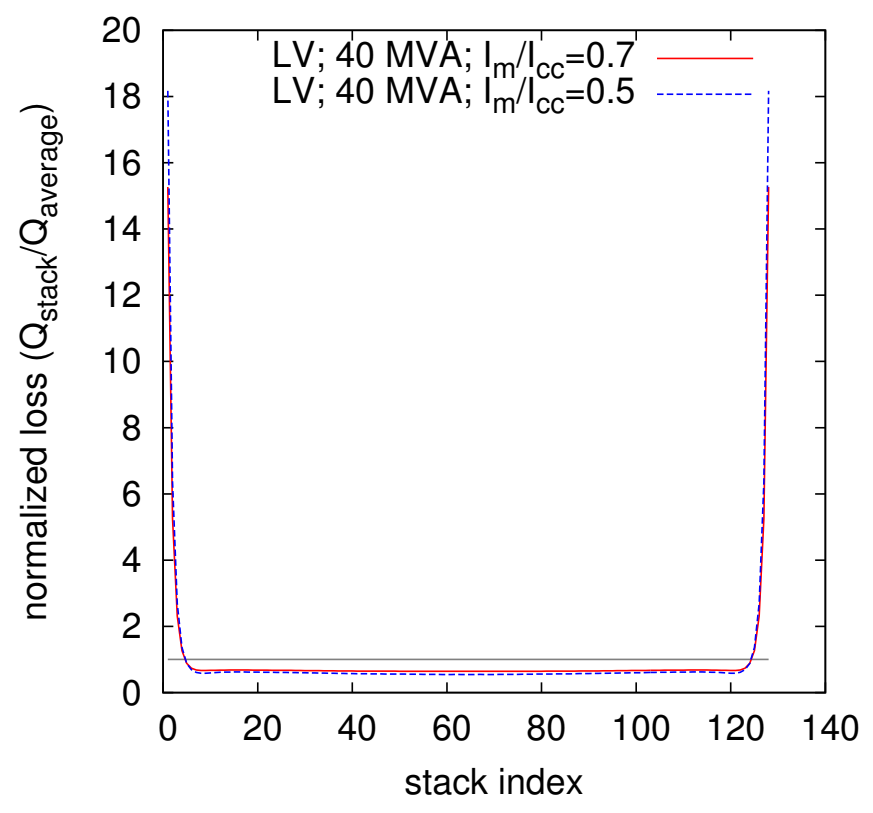

Figure 25: The normalized AC loss in the $\mathrm{LV}$ winding $\left(Q_{\text {stack }}\right.$ and $Q_{\text {average }}$ are the loss per cycle and cable length in one the of the stacks of the Roebel cable and the average in all stacks, respectively) is the highest at the end turns. In addition, there is a substantial AC loss contribution from the central turns. The horizontal gray line represents the average value. 


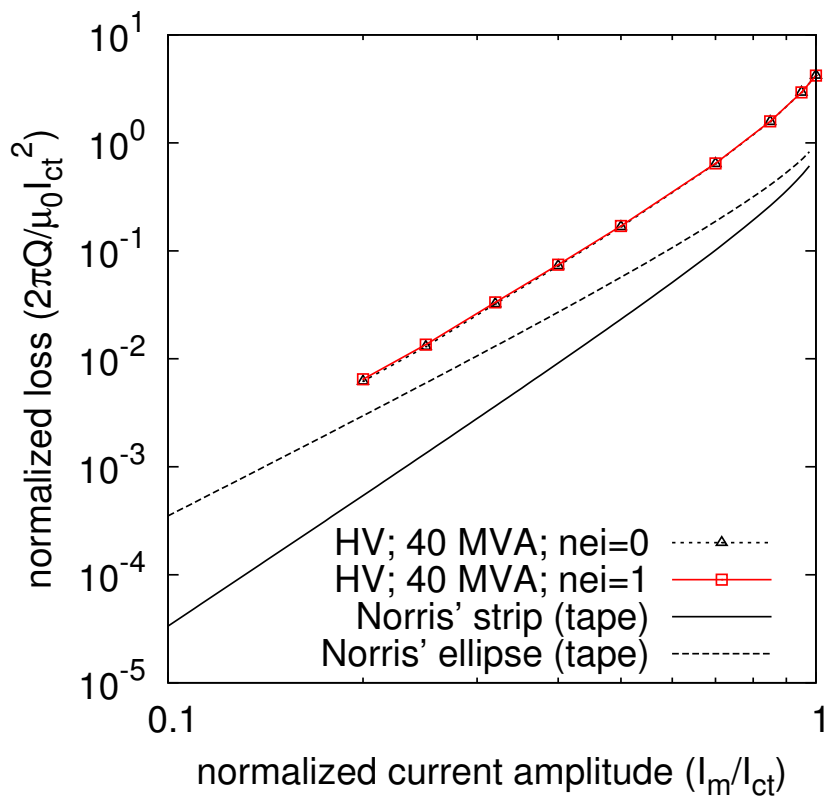

Figure 26: The normalized AC loss in the HV winding (with LV in short circuit) of the 40 MVA transformer is larger than the Norris' strip line 32 for a single tape. The normalized loss is $2 \pi Q /\left(\mu_{0} I_{c}^{2}\right)$, where $Q$ is the loss per cycle and unit tape length. $I_{m}$ is the AC current amplitude. Curves for "nei=1" and "nei=0" are for the neighbor approximations of the first and zeroth order, respectively. 


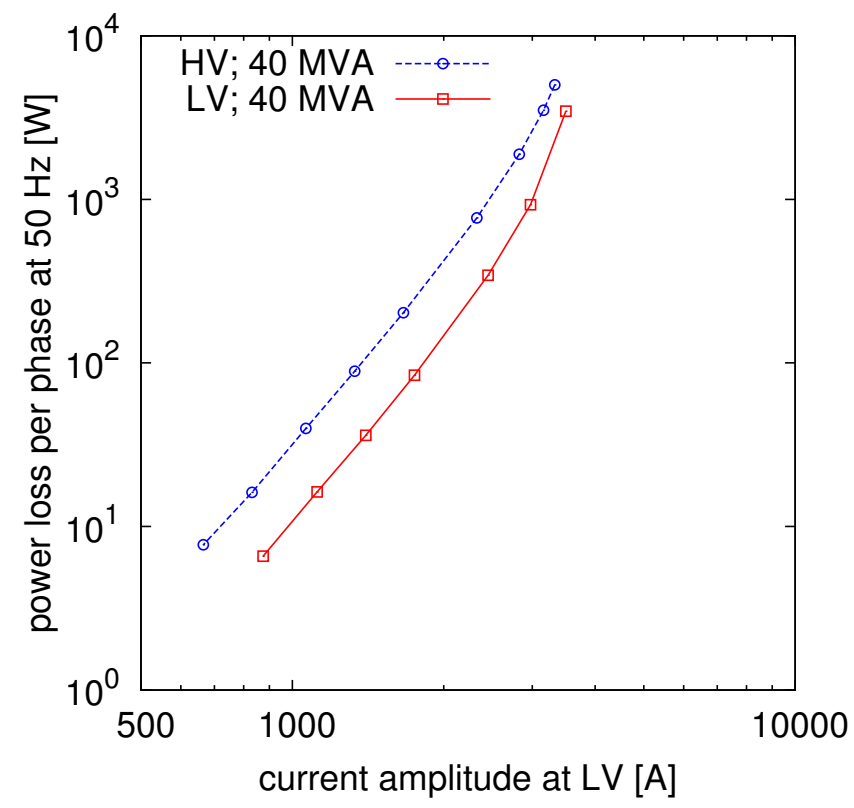

Figure 27: For the same fraction of $\mathrm{AC}$ current relative to the critical current, the $\mathrm{AC}$ loss in the $\mathrm{HV}$ and $\mathrm{LV}$ windings are of the same order of magnitude (the plotted $\mathrm{AC}$ loss is for one of the phases of the three-phase transformer). These calculations assume a critical current of the superconducting tapes of $486 \mathrm{~A} / \mathrm{cm}$, corresponding to 3500 A critical current for the Roebel cable in the LV winding. With this data, the AC loss in each winding is below $2.5 \mathrm{~kW}$ per phase at $50 \mathrm{~Hz}$ and the rated current for the LV winding (2969 A). 


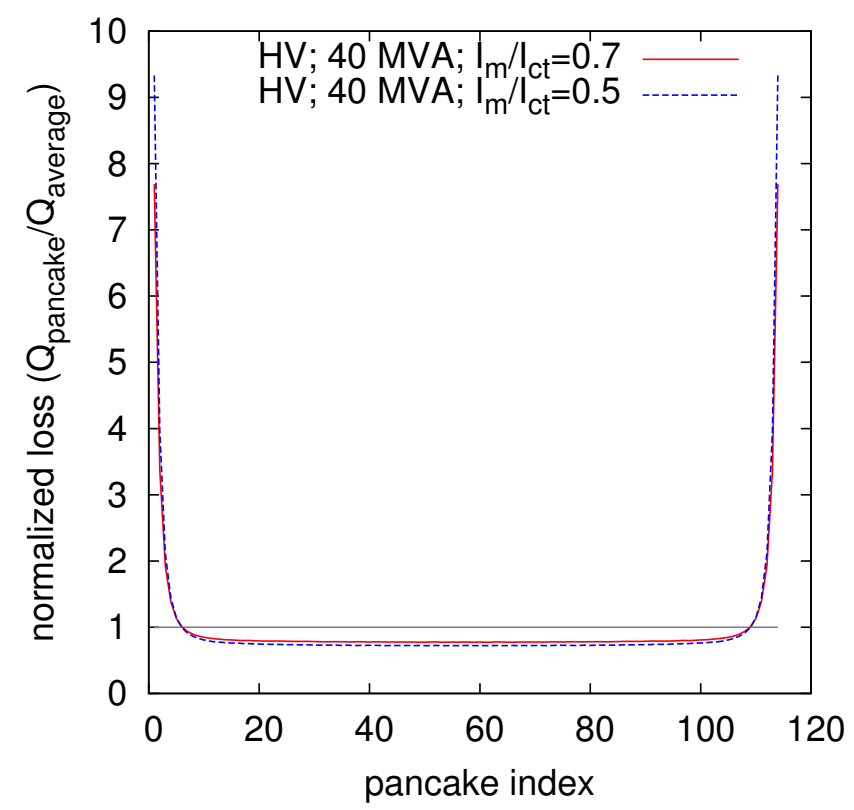

Figure 28: Although the AC loss in the top and bottom pancakes of the HV winding is much larger than the average, most of the AC loss is generated by the pancakes away from the ends.

central pancakes is the main contribution to the AC loss. In particular, the $\mathrm{AC}$ loss at the central turns is 77 and $72 \%$ of the average and the AC loss due to the first four pancakes closer to each end account for $25 \%$ and $30 \%$ of the total one at current amplitudes 70 and $50 \%$ of the critical current, respectively.

The average radial magnetic field in each pancake (or stack of strands in the LV) in figure 29 reveals that the average radial magnetic field in the $\mathrm{HV}$ is higher than in the LV for axial positions away from the ends (1.9 times higher field in the HV than in the LV). The cause of this higher radial magnetic field is mainly the higher axial separation between turns in the HV. In order to confirm this, we calculated the case of a HV winding with the same axial separation as for the LV $(1 \mathrm{~mm})$ and reducing the whole transformer height accordingly (both $\mathrm{HV}$ and associated LV, with new total height $569 \mathrm{~mm}$ ). As expected, in the HV the average radial magnetic field at the turns away from the ends is practically the same as in the original LV (figure 29).

Therefore, the AC loss may be reduced by reducing the gap between pancakes, which may be done by either increasing the number of pancakes (and reducing the number of turns per pancake) or increasing the tape width. However, there may be electrical isolation issues due to the high voltage of the winding. 


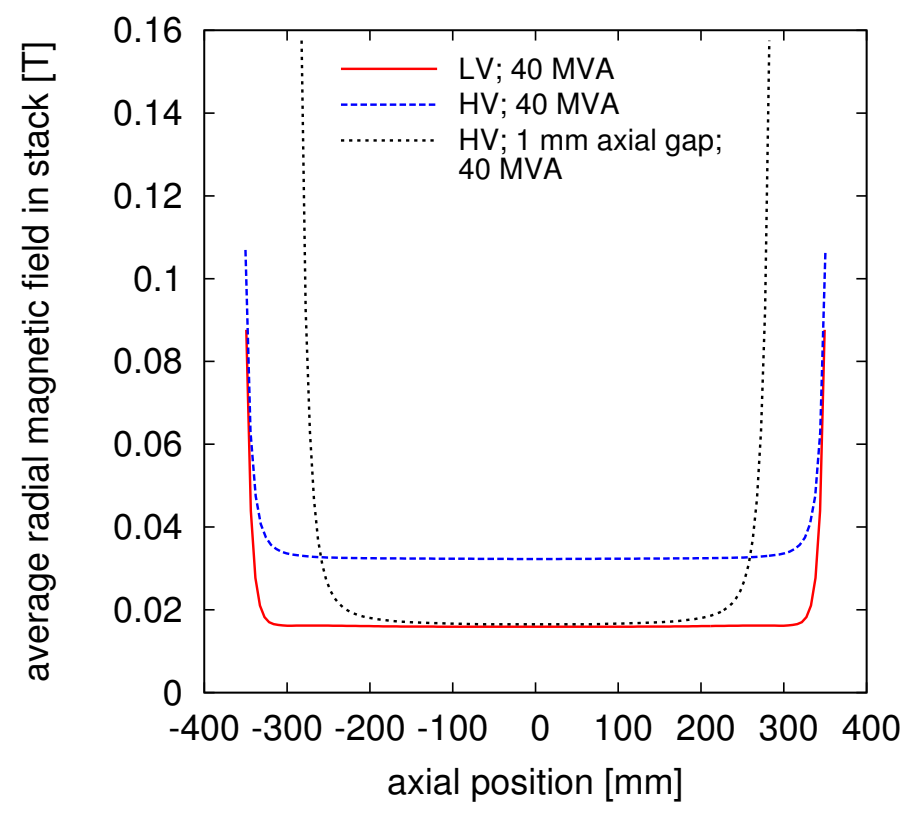

Figure 29: Average radial magnetic field in each stack or pancake coil of the LV and $\mathrm{HV}$ windings, respectively. At the central turns, the average radial field in the $\mathrm{HV}$ winding is much higher than in the LV. Using the same axial gap in the HV $(1 \mathrm{~mm})$ as in the LV, results in practically the same average radial field in the central turns. All calculations are for $I_{m}=0.7 I_{c}$ in the $\mathrm{LV}$ winding and assume constant $J_{c}$. 


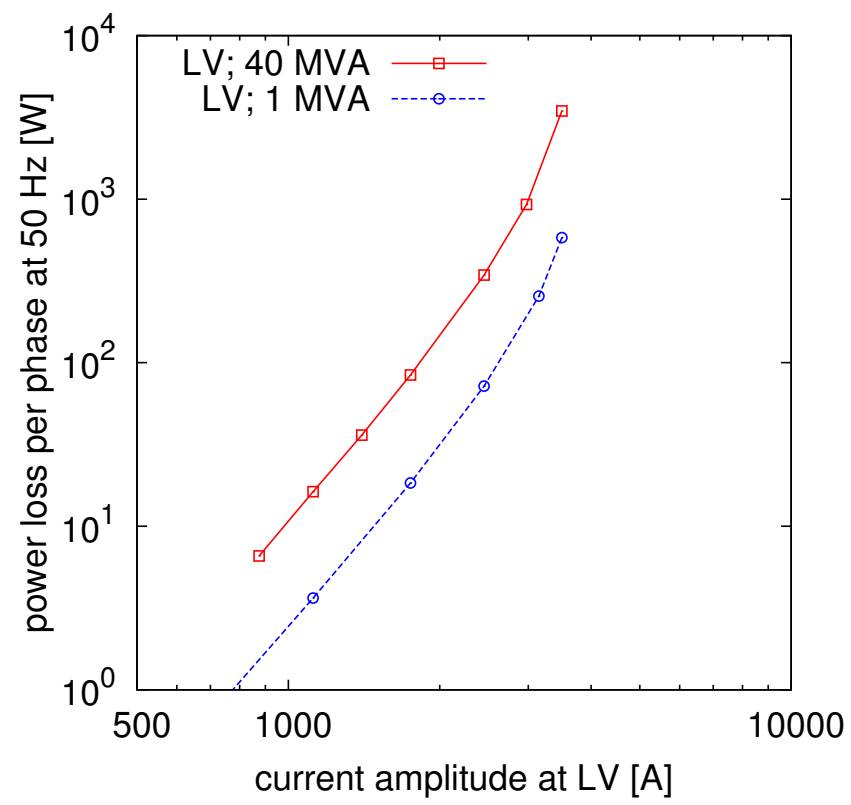

Figure 30: Compared with the 1 MVA transformer, the AC loss in the LV of the 40 MVA increases by only a factor around 4.9 and 4.6 at 85 and $50 \%$ of the current capacity, respectively. The computations assume $3500 \mathrm{~A}$ critical current of the Roebel cable for both cases.

\subsection{AC loss comparison between the 1 MVA and 40 MVA transformers}

This section compares the AC loss of the 40 MVA and 1 MVA transformers. However, the reader should keep in mind that the rated power is not the only factor that influences the AC loss, since the transformers differ in several other aspects (see table 1). For the sake of comparison, we assume that the critical current per tape width is the same in both transformers. We take a value of $486 \mathrm{~A} / \mathrm{cm}$, corresponding to $3500 \mathrm{~A}$ critical current for the Roebel cable in the LV winding. This is not an unachievable critical current using commercially available conductor. For Fujikura tapes [44, this critical current at the magnetic fields in the transformer, around $400 \mathrm{mT}$, are achieved at $65 \mathrm{~K}$, based on the measured critical current density with perpendicular applied field.

The AC loss in the LV winding scales from the $1 \mathrm{MVA}$ to the $40 \mathrm{MVA}$ transformer only by a factor of around 4.9 and 4.6 at 85 and $50 \%$ of the current capacity, respectively (see figure 30 .

Regarding the HV winding, the scaling comparison is less straightforward. Comparing with the same current amplitude in the HV winding, scaling from 1 MVA to 40 MVA only increases the loss by a factor around 1.75 at $50 \%$ of the critical current (see figure 31). However, comparing with the same current in the LV winding, upscaling increases the AC loss by one order of magnitude 


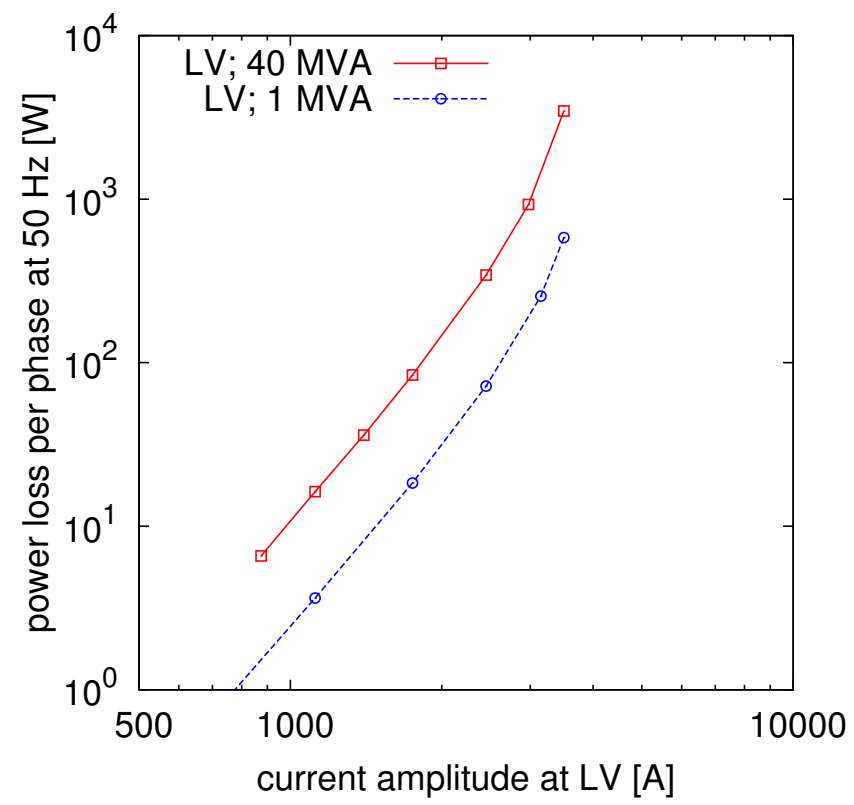

Figure 31: When comparing for the same current amplitude in the HV winding for the $1 \mathrm{MVA}$ and $40 \mathrm{MVA}$ transformers, the AC loss in the HV winding of the $40 \mathrm{MVA}$ transformer increases by only a factor around 1.75 at $50 \%$ of the current capacity.

(see figure 32). This kind of comparison is more useful to determine the scaling of the $\mathrm{AC}$ loss for the whole transformer. The reason for these very different scaling factors is that for the same $I_{m} / I_{c}$ in the LV winding, the $I_{m} / I_{c}$ ratio in the $\mathrm{HV}$ is lower for the $1 \mathrm{MVA}$ transformer than for the $40 \mathrm{MVA}$ transformer (0.38 and 1.27 , respectively).

As a result, the total loss in the 40 MVA transformer is between 12.5 and 16.1 times higher than for the 1 MVA one (see figure 33). This is still much lower than the ratio 40 of rated power.

\subsection{AC loss dependence with critical current}

The AC loss at the rated current of the LV winding (LV winding in short circuit) decreases with decreasing tape critical current (see figure 34). To achieve a load loss equivalent to to that of a typical 40 MVA transformer assuming a cooling system with a cooling penalty, defined as the inverse of the coefficient of performance 1/COP, of 30, typical of Gifford-McMahon cryocoolers at $65 \mathrm{~K}$, the AC loss at $50 \mathrm{~Hz}$ per phase should not be higher than $1.5 \mathrm{~kW}$ [45]. This goal is achieved at a tape critical current of $656 \mathrm{~A} / \mathrm{cm}$. Since $110 \mathrm{~m}$ long samples with $I_{c}$ above $700 \mathrm{~A} / \mathrm{cm}$ in $77 \mathrm{~K}$ and self-field have been already produced by Fujikura [46], tapes with this specification may be commercially available in the near future. 


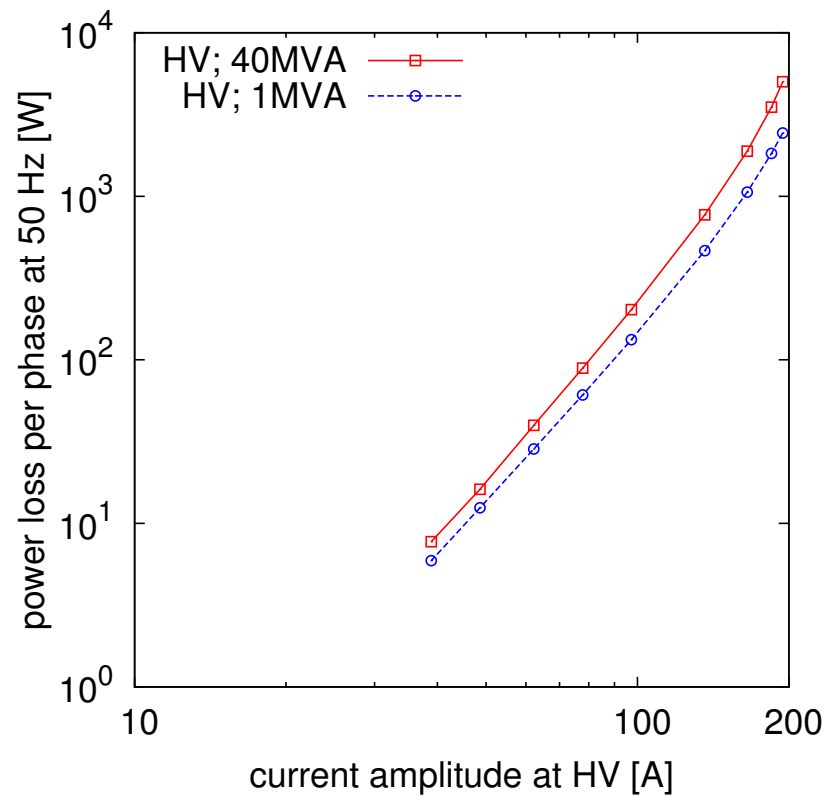

Figure 32: When comparing for the same current amplitude at the LV winding (more relevant for the full transformer), the AC loss in the $\mathrm{HV}$ winding scales by around a factor 10 for the same current in the LV winding.

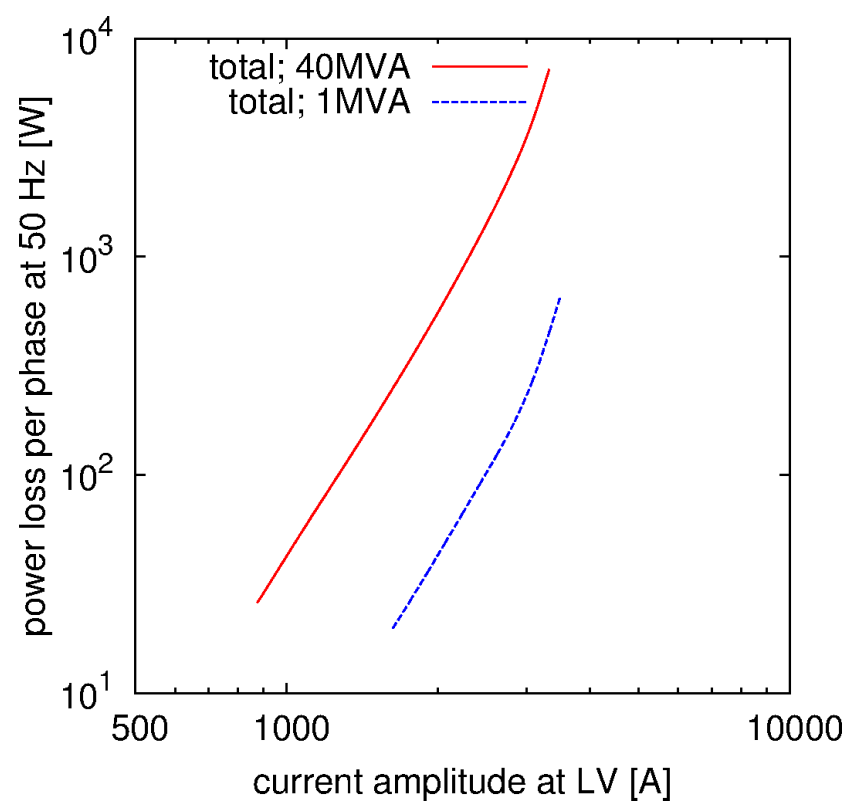

Figure 33: Comparing the 1 MVA and 40 MVA transformers, the total loss scales by a factor between 12.6 and 16.1 for the same current in the LV winding. 


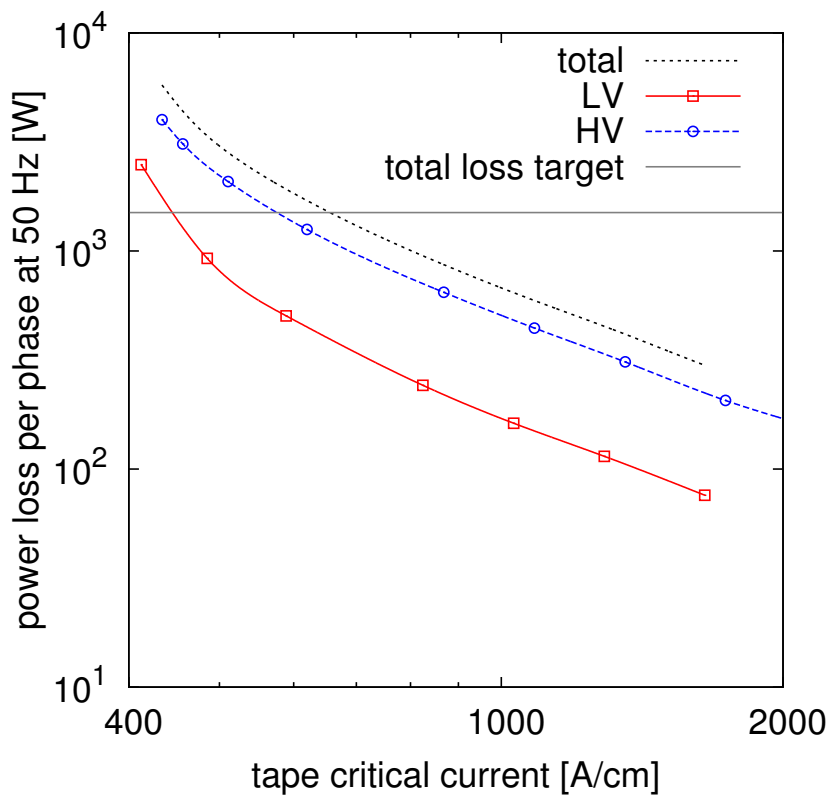

Figure 34: The AC loss in the 40 MVA transformer at the rated current of the LV winding (2969 A amplitude) decreases with decreasing the tape critical current (LV winding in short circuit). In order to achive the target of $1.5 \mathrm{~kW}$ of total AC loss, a tape critical current of $656 \mathrm{~A} / \mathrm{cm}$ is necessary. 


\section{Conclusion}

This article presented modelling and measurement results for the AC loss in fully superconducting transformers with Roebel-cable solenoids as low-voltage (LV) windings. In particular, a $1 \mathrm{MVA} 11 \mathrm{kV} / 415 \mathrm{~V}$ 3-phase prototype has been both measured and modelled. The measurements were made by electrical means and the calculations used the Minimum Magnetic Energy Variation Method 9.10. They showed good agreement. This supports the validity of extending the modelling to a 40 MVA $110 \mathrm{kV} / 11 \mathrm{kV} 3$-phase transformer design. Of special relevance is the fact that this 40 MVA transformer contains around 1000 turns or strands in the high-voltage (HV) and LV windings, respectively. In addition, we have also studied a stand-alone Roebel solenoid, since that situation had not been not previously modelled.

Computations revealed that the $\mathrm{AC}$ loss in the $\mathrm{LV}$ winding is much lower when it is inserted in the transformer than as stand-alone solenoid. This is caused by the fact that the radial field created by the HV winding partially cancels the one from the LV. This loss reduction also applies for the HV.

For the 1 MVA transformer, we have found that the AC loss is dominated by the LV winding. We also found that the calculations for either constant $J_{c}$ or magnetic-field dependent $J_{c}$ agree with the experiments at hight current amplitudes, including the rated current. Discrepancies at lower current amplitudes may be caused by eddy currents in the copper terminals of the LV winding.

For the 40 MVA transformer, we have found that the AC loss is dominated by the HV winding, which a the rated current is around twice as large as the $\mathrm{LV}$ loss. Although the local loss at the 4 pancakes of the $\mathrm{HV}$ closer to each end is much higher than the average in the whole winding, $70 \%$ of the loss is generated at the rest of the winding at $0.5 I_{c}$. For the $L V$, the situation is qualitatively similar but the part excluding the 2 Roebel cable turns closer to each end contributes $55 \%$ the loss. The loss as a fraction of rated power of this transformer is between 0.32 and 0.40 of that for the 1 MVA transformer, hence with increasing rated power the efficiency increases. For the 40 MVA device, the target total loss is $1.5 \mathrm{~kW}$ per phase 45 . This could be achieved with a minimum tape critical current of $656 \mathrm{~A} / \mathrm{cm}$ at $65 \mathrm{~K}$, a critical current that should be commercially available in the near future.

This work has shown that Roebel cables can be used as high-current conductors in LV windings. We have shown that the modelling tool in this work can reliably predict the AC loss in real power applications, even when they contain superconducting windings of thousands of turns.

\section{Acknowledgements}

This study was supported by the New Zealand Ministry of Business, Innovation and Employment under contract C08X1301. 


\section{A Relation between currents in the $\mathrm{HV}$ and $\mathrm{LV}$ windings}

The relation between the currents in the HV and LV windings, $I_{H V}(t)$ and $I_{L V}(t)$ respectively, are calculated as follows. The mutual inductance between windings, $M$, is calculated numerically assuming uniform current distribution in the superconducting layers of the winding in which the $\mathrm{AC}$ loss is being calculated and in the whole cross-section of the other winding (the reader may find more details in B). Assuming that both the LV and $\mathrm{HV}$ windings are ideal inductances, the relation between the current in the primary and secondary windings is

$$
\tilde{I}_{H V}(\omega)=-\frac{L_{L V}}{M}\left(1-\frac{i R}{\omega L_{L V}}\right) \tilde{I}_{L V}(\omega),
$$

where $R$ is a load resistance connected across the LV winding (in this report it is zero), $M, L_{L V}$ are the mutual- and self- inductances of the LV winding, $\tilde{I}_{H V}(\omega)$

and $\tilde{I}_{L V}(\omega)$ are the Fourier transforms of $I_{H V}(t)$ and $I_{L V}(t)$, respectively, $\omega$ is the angular frequency and $i$ is the imaginary unit. The equation above has been used to calculate the current amplitude and phase of one winding, given a sinusoidal current in the other winding. In particular, the relation between amplitudes is

$$
I_{m, L V}=I_{m, H V} \frac{M}{\sqrt{\left(\frac{R}{\omega}\right)^{2}+L_{L V}^{2}}},
$$

where $I_{m, H V}, I_{m, L V}$ are the current amplitudes in the HV and LV windings, respectively. The phase difference between the HV and LV windings, $\theta_{H V}-\theta_{L V}$, follows

$$
\theta_{H V}-\theta_{L V}=\arg \left(-1+\frac{i R}{\omega L_{L V}}\right)
$$

the function arg being the argument of a complex number. Consistently, the equation above predicts that the current in both windings is in phase opposition when the LV winding is in short circuit $(R=0)$.

\section{B Calculation of self and mutual inductances}

This section details the calculation method for the mutual, $M$, and self inductances, $L_{L V}$ and $L_{H V}$ in the LV and $\mathrm{HV}$ windings, respectively. The text below is for the case that the AC loss in the $\mathrm{HV}$ winding is calculated; and thence, the detailed current density with magnetization currents is only modelled in that winding. The calculation for the AC loss in the LV winding is analogous, just replacing HV by LV and opposite in the explanation below. Actually, the obtained values of $M, L_{L V}$ and $L_{H V}$ for the AC loss calculations of either $\mathrm{HV}$ and LV do not differ within the given 3-digit accuracy in table 4

The calculation of $L_{L V}$ is done numerically by dividing the cross section of the LV winding in rings of rectangular cross-section and assuming that the 
current flows in the center of the elements. The number of elements in the radial and axial directions, $n_{r}$ and $n_{z}$ respectively, are chosen in such a way that the elements are as square as possible, given a certain number of elements in the axial direction. In particular, $n_{r}=\operatorname{int}\left(n_{z} h_{r} / h_{z}+1 / 2\right)$, where $h_{r}$ and $h_{z}$ are the coil dimensions in the radial and axial dimensions, respectively. Then, the vector potential is calculated at the centre of all the elements by means of the formula of the vector potential generated by a thin wire (page 112 of [47). Afterwards, $L_{L V}$ is calculated as $L_{L V}=2 U / I^{2}$, where $U$ is the coil magnetic energy $U=\pi \int_{S} r J A \mathrm{~d} S$, where $S$ is the winding cross-section, $r$ is the radial coordinate, $J$ is the engineering current density and $A$ is the vector potential. The computation routine increases the number of elements until the result converges.

The value of the self-inductance of the winding for which the AC loss is calculated (in this case the HV winding) is computed by assuming uniform current density in the superconducting turns or strands and using the same element division as for the Minimum Magnetic Energy Variation method, which already takes the self-interaction term into account [9].

Finally, the mutual inductance $M$ is calculated by assuming uniform current density in the engineering cross-section of the LV winding and in the superconducting layer of the tapes in the HV winding. The discretization of the LV winding was such that the separation between elementary rings was not larger than half the gap between windings. Numerical simulations with increasing number of elements in the LV winding showed that finer meshes do not modify the result.

\section{References}

\section{References}

[1] X. Obradors and T. Puig. Coated conductors for power applications: materials challenges. Supercond. Sci. Technol., 27(4):044003, 2014.

[2] Y. Zhang, T. F. Lehner, T. Fukushima, H. Sakamoto, and D. W. Hazelton. Progress in production and performance of second generation (2G) HTS wire for practical applications. IEEE Trans. Appl. Supercond., 24(5):1-5, 2014.

[3] O. Tsukamoto. Overview of superconductivity in Japan-Strategy road map and R\&D status. Physica C, 468(15):1101-1111, 2008.

[4] F. Grilli, E. Pardo, A. Stenvall, D. N. Nguyen, W. Yuan, and F. Gömöry. Computation of losses in HTS under the action of varying magnetic fields and currents. IEEE Trans. Appl. Supercond., 24(1):8200433, 2014.

[5] A. M. Wolsky. HTS from precommercial to commercial: A roadmap to future use of HTS by the power sector. International Energy Agency, 2013. Available at http://superconductivityiea.rse-web.it. 
[6] M. P. Staines, Z. Jiang, N. Glasson, R. G. Buckley, and M. Pannu. Hightemperature superconducting (HTS) transformers for power grid applications. In Superconductors in the Power Grid: Materials and Applications. Woodhead Publishing Series in Energy, 2015. Chapter 12. ISBN 10: 1782420290 .

[7] E. Pardo and F. Grilli. Numerical simulations of the angular dependence of magnetization ac losses: coated conductors, roebel cables and double pancake coils. Supercond. Sci. Technol., 25:014008, 2012.

[8] F. Grilli and S. P. Ashworth. Measuring transport ac losses in YBCOcoated conductor coils. Supercond. Sci. Technol., 20:794, 2007.

[9] E. Pardo. Modeling of coated conductor pancake coils with a large number of turns. Supercond. Sci. Technol., 21:065014, 2008.

[10] E. Pardo, J. Šouc, and J. Kováč. AC loss in ReBCO pancake coils and stacks of them: modelling and measurement. Supercond. Sci. Technol., 25:035003, 2012.

[11] E. Pardo, J. Šouc, and L. Frolek. Electromagnetic modelling of superconductors with a smooth current-voltage relation: variational principle and coils from a few turns to large magnets. Supercond. Sci. Technol., 28:044003, 2015.

[12] L. Prigozhin and V. Sokolovsky. Computing AC losses in stacks of hightemperature superconducting tapes. Supercond. Sci. Technol., 24:075012, 2011.

[13] M.D. Ainslie, V.M. Rodriguez-Zermeno, Z. Hong, W. Yuan, T.J. Flack, and T.A. Coombs. An improved FEM model for computing transport AC loss in coils made of RABiTS YBCO coated conductors for electric machines. Supercond. Sci. Technol., 24:045005, 2011.

[14] VM Rodriguez-Zermeno, N. Mijatovic, C. Traeholt, T. Zirngibl, E. Seiler, AB Abrahamsen, NF Pedersen, and MP Sorensen. Towards faster FEM simulation of thin film superconductors: A multiscale approach. IEEE Trans. Appl. Supercond., 21(3):3273-3276, 2011.

[15] M. Zhang, W. Yuan, D. K. Hilton, M. D. Canassy, and U. P. Trociewitz. Study of second-generation high-temperature superconducting magnets: the self-field screening effect. Supercond. Sci. Technol., 27(9):095010, 2014.

[16] E. Pardo. Calculation of AC loss in coated conductor coils with a large number of turns. Supercond. Sci. Technol., 26(10):105017, 2013.

[17] V. M. R. Zermeno, A. B. Abrahamsen, N. Mijatovic, B. B. Jensen, and M. P. Sørensen. Calculation of alternating current losses in stacks and coils made of second generation high temperature superconducting tapes for large scale applications. J. Appl. Phys., 114(17):173901, 2013. 
[18] F. Grilli and E. Pardo. Simulation of ac loss in Roebel coated conductor cables. Supercond. Sci. Technol., 23:115018, 2010.

[19] M. Nii, N. Amemiya, and T. Nakamura. Three-dimensional model for numerical electromagnetic field analyses of coated superconductors and its application to roebel cables. Supercond. Sci. Technol., 25(9):095011, 2012.

[20] V. M. R. Zermeno, F. Grilli, and F. Sirois. A full 3D time-dependent electromagnetic model for Roebel cables. Supercond. Sci. Technol., 26(5):052001, 2013.

[21] N. Amemiya, T. Tsukamoto, M. Nii, T. Komeda, T. Nakamura, and Z. Jiang. Alternating current loss characteristics of a Roebel cable consisting of coated conductors and a three-dimensional structure. Supercond. Sci. Technol., 27:035007, 2014.

[22] F. Grilli, V. M. R. Zermeno, E. Pardo, M. Vojenciak, J. Brand, A. Kario, and W. Goldacker. Self-field effects and AC losses in pancake coils assembled from coated conductor roebel cables. IEEE Trans. Appl. Supercond., 24(3):4801005, 2014.

[23] N. Glasson, M. Staines, R. Buckley, M. Pannu, and S. Kalsi. Development of a 1 MVA 3-phase superconducting transformer using YBCO Roebel cable. IEEE Trans. Appl. Supercond., 21(3):1393-1396, 2011.

[24] M. Staines, N. Glasson, M. Pannu, K. P. Thakur, R. Badcock, N. Allpress, P. D'Souza, and E. Talantsev. The development of a Roebel cable based 1 MVA HTS transformer. Supercond. Sci. Technol., 25(1):014002, 2012.

[25] N. D. Glasson, M. P. Staines, Z. Jiang, and N. S. Allpress. Verification testing for a 1 MVA 3-phase demonstration transformer using 2G-HTS Roebel cable. IEEE Trans. Appl. Supercond., 23(3):5500206-5500206, 2013.

[26] SuperPower, Inc. http://www.superpower-inc.com/.

[27] Z. Jiang, M. Staines, N. J. Long, R. A. Badcock, C. Bumby, E. Talantsev, K. Hamilton, R. G. Buckley, and N. Amemiya. The scaling of transport AC losses in Roebel cables with varying strand parameters. Supercond. Sci. Technol., 27(7):075007, 2014.

[28] O. Tsukamoto. AC losses in a type II superconductor strip with inhomogeneous critical current distribution. Supercond. Sci. Technol., 18:596, 2005.

[29] Z. Jiang, N. Amemiya, O. Maruyama, and Y. Shiohara. Critical current density distribution and magnetization loss in YBCO coated conductors. Physica C, 463:790-794, 2007.

[30] M. Solovyov, E. Pardo, J. Šouc, F. Gömöry, M. Skarba, P. Konopka, M. Pekarčíková, and J. Janovec. Non-uniformity of coated conductor tapes. Supercond. Sci. Technol., 26(11):115013, 2013. 
[31] S. Terzieva, M. Vojenčiak, E. Pardo, F. Grilli, A. Drechsler, A. Kling, A. Kudymow, F. Gömöry, and W. Goldacker. Transport and magnetization ac losses of ROEBEL assembled coated conductor cables: measurements and calculations. Supercond. Sci. Technol., 23:014023, 2010.

[32] W. T. Norris. Calculation of hysteresis losses in hard superconductors carrying ac: isolated conductors and edges of thin sheets. J. Phys. D: Appl. Phys., 3:489-507, 1970.

[33] Z. Jiang, N. J. Long, M. Staines, Q. Li, R. A. Slade, N. Amemiya, and A. D. Caplin. Transport AC loss measurements in single-and two-layer parallel coated conductor arrays with low turn numbers. IEEE Trans. Appl. Supercond., 22(6):8200306-8200306, 2012.

[34] M. Staines and Z. Jiang. Measurement of AC loss in individual turns of an hts solenoid. Physics Procedia, 36:859-865, 2012.

[35] E. Pardo, D.-X. Chen, A. Sanchez, and C. Navau. Alternating current loss in rectangular superconducting bars with a constant critical-current density. Supercond. Sci. Technol., 17:83-87, 2004.

[36] J. R. Clem. Field and current distributions and ac losses in a bifilar stack of superconducting strips. Phys. Rev. B, 77:134506, 2008.

[37] F. Grilli, R. Brambilla, L. F. Martini, F. Sirois, D. N. Nguyen, and S. P. Ashworth. Current density distribution in multiple YBCO coated conductors by coupled integral equations. IEEE Trans. Appl. Supercond., 19(3):2859-2862, 2009.

[38] D. N. Nguyen, J. Y. Coulter, J. O. Willis, S. P. Ashworth, H. P. Kraemer, W. Schmidt, B. Carter, and A. Otto. AC loss and critical current characterization of a noninductive coil of two-in-hand RABiTS YBCO tape for fault current limiter applications. Supercond. Sci. Technol., 24:035017, 2011.

[39] J. Šouc, F. Gömöry, and M. Vojenčiak. Coated conductor arrangement for reduced AC losses in a resistive-type superconducting fault current limiter. Supercond. Sci. Technol., 25:014005, 2012.

[40] O. Nackel and M. Noe. Conceptual design study of an air coil fault current limiter. IEEE Trans. Appl. Supercond., 23(3):5602404, 2013.

[41] H. Heydari, A. A. Abrishami, and M. M. Bidgoli. Comprehensive analysis for magnetic shield superconducting fault current limiters. IEEE Trans. Appl. Supercond., 23(5):5604610-5604610, 2013.

[42] H. Heydari, F. Faghihi, and R. Aligholizadeh. A new approach for AC loss reduction in HTS transformer using auxiliary windings, case study: $25 \mathrm{kA}$ HTS current injection transformer. Supercond. Sci. Technol., 21:015009, 2008. 
[43] Y. B. Kim, C. F. Hempstead, and A. R. Strnad. Critical persistent currents in hard superconductors. Phys. Rev. Lett., 9(7):306-309, 1962.

[44] Fujikura, http://www.fujikura.com/solutions/superconductingwire/.

[45] M. Staines, L. Jolliffe, A. Hunze, Pannu M., Pardo E., and Glasson N. Will we see superconducting transformers in the grid anytime soon? EEA Conference ES Exhibition. Preprint.

[46] Y. Iijima. High-performance Y-based superconducting wire and their applications. Fujikura Technical Review, 42, 2013. Available at www.fujikura.co.jp/eng/rd/gihou/backnumber.

[47] L. D. Landau, E. M. Lifshitz, and L. P. Pitaevskii. Electrodynamics of Continuous Media. Elsevier Butterworth Heinemann, Amsterdam, 2008. 